\title{
Polarization and movement of keratocytes: a multiscale modelling approach
}

\author{
Athanasius F. M. Marée ${ }^{1}$, Alexandra Jilkine ${ }^{2}$, Adriana Dawes ${ }^{2}$, \\ Verônica A. Grieneisen ${ }^{1}$, and Leah Edelstein-Keshet ${ }^{2}$
}

27th March 2006

\author{
${ }^{1}$ Theoretical Biology/Bioinformatics, Utrecht University, Utrecht, the Netherlands \\ ${ }^{2}$ Department of Mathematics, University of British Columbia, Vancouver, B.C., Canada, V6T $1 Z 2$
}

Lee A. Segel (2001):“... A simulation of a developing organism must represent the motion of a large number of interacting cells. Like other materials, cells respect the laws of physics. Each cell is not only driven by external forces but also generates internal forces by assembling and disassembling an ephemeral cytoskeleton under the direction of various controlling chemicals. A further complication is that although cell shape depends on the resultant of all of the forces, the forces themselves depend on the shape..."

\begin{abstract}
Eukariotic cell motility is a complex phenomenon, in which the cytoskeleton and its major constituent, actin, play an essential role. Actin forms polymers of long, stiff filaments that are cross-linked into an anisotropic network inside a thin sheet-like cellular protrusion, the lamellipod. At the leading edge of this structure, polymerization of actin filaments creates the force that pushes out the membrane and leads to translocation of a motile cell. Dynamics of the actin network account for changes in cell shape, crawling motion and turning of the cell in response to external cues. Regulating the dynamics of the cytoskeleton, and playing a central role in signal transduction in the cell, are Cdc42, Rac and Rho (GTPases of the rho family, collectively known as the small G-proteins) and the actin nucleating complex, Arp2/3.

In this paper, we use a multiscale modelling approach in a 2D model of a motile cell. We describe the mutual interactions of the small G-proteins, and their effects on capping and sidebranching of actin filaments. We incorporate the pushing exerted by oriented actin filament ends on the cell edge, and a Rho-dependent contraction force. Combining these biochemical and mechanical aspects, we investigate the dynamics of a model epidermal fish keratocyte through in silico experiments. Our model gives insight into how, in response to some cue, a cell can polarize, form a leading edge, and move; concomitantly it explains how a keratocyte cell can maintain its shape and polarity, even after removal of the initial stimulus, and how it
\end{abstract}


can change direction quickly in response to changes in its environment. We show that establishment of polarity stems from interactions of $\mathrm{Cdc} 42$, Rac and Rho, while maintenance and robustness of polarity is due to the rapid cytosolic diffusion of the inactive (GDI-bound) forms of the small G-proteins. Our model produces cell shape that closely resembles the keratocytes and correct speeds for biologically reasonable parameter values. Movies of the simulations can be obtained from http://theory.bio.uu.nl/stan/keratocyte.

\section{Introduction}

Cell motility plays an important role in embryonic development, immune surveillance, wound healing, and many other cellular functions. As demonstrated by the opening quotation, Lee Segel realized that the outstanding complexity in the movement of cells, is due partly to feedbacks at all levels. Following his chain of thoughts, our aim is to show that these feedbacks indeed exist between the cytoskeleton, the chemical signals controlling it and the cell shape: moreover, we will argue that this entanglement is essential for cell motility. Given the empirical information collected throughout the years at each specific level, the time is ripe to integrate these levels into a single modelling framework with multi-level interactions.

After many decades (Abercrombie, 1980) and many cell types studied (neurons (Krewson et al., 1994; Neely and Nicholls, 1995); neutrophils (Ehrengruber et al., 1996; Takubo and Tatsumi, 1997); fibroblasts (Wells et al., 1998; Kole et al., 2005); etc.), it is still to be understood how cells can polarize and then accurately and robustly regulate their motion in response to environmental cues. Our paper addresses these questions, by acknowledging that the factors that give rise to regulated cell motion are distributed over multiple spatial and temporal levels. Two aspects of our approach should be emphasized here. First, in order to include multiple levels without obscuring the underlying mechanism responsible for cell motility phenomena, we have to use a concise description. This means that we focus on the essential biological agents and their interactions, necessarily leaving out many specific details. Second, by assembling these essential pieces and showing that they can already account for the complex biologically relevant behaviour, we aim to prove that these constitute a sufficient set of "minimal biological requirements" for cell motility behaviour.

\subsection{Actin cytoskeleton}

The dominant force in front-edge protrusion of a motile cell is the actin cytoskeleton (Cramer et al., 1994; Abraham et al., 1999). Actin filaments have two distinct ends, with different affinities for monomers. The "barbed" (or plus) ends favour actin monomer addition, and (in the absence of physical impediments such as a membrane) ordinarily grow rapidly, given cytoplasmic levels of free monomeric actin. Barbed ends of actin filaments are generally oriented towards the leading edge of the cell (Svitkina et al., 1997; Pollard and Borisy, 2003); they provide the polymerization force that pushes the membrane outwards. The "pointed" (or minus) ends of actin filaments are generally concentrated further back into the cell, where actin disassembly dominates over assembly. (Actin filament growth at the front and loss at the back is called "treadmilling": the filaments are largely stationary save for very slow retrograde flow.) Together, these actin filaments are networked and cross-linked to form a mesh that gives the cell its characteristic shape and structure. 
The actin filament network is dynamic, undergoing remodelling and constantly extending or retracting, based on signals received by the cell from its surroundings: an external gradient, e.g. of a chemoattractant, processed and integrated internally in a motile cell and transduced to its cytoskeleton, eventually determines the direction of cell movement (Haugh et al., 2000; Funamoto et al., 2002). Signal transduction pathways transmit signals from membrane-bound receptors to 'central nodes', including the small G-proteins, Cdc42, Rac, and Rho (also called the "rho family proteins") (Meili and Firtel, 2003). Their activity, in turn, impinges directly on the actin dynamics (see Fig. 1), regulating and fine-tuning the polymerization, disassembly, and nucleation of filaments, as well as capping and uncapping of actin filament barbed ends.

A paradigm system for actin based cell motility is the fish epidermal keratocyte (Svitkina and Borisy, 1999; Ream et al., 2003), a cell that moves smoothly, (with speed circa $0.25 \mu \mathrm{m} / \mathrm{s}$ (Laurent et al., 2005)) while retaining a typical crescent shape. This mode of cell motion is not restricted to keratocytes, as other cell lineages can present this behaviour as well (see Discussion). Remarkably, even well-prepared fragments of a keratocyte, lacking nuclei and organelles, can, given a transient mechanical stimulus, initiate and sustain such 'gliding' motility (Verkhovsky et al., 1999). Although not all details of the biochemistry are known, this robustness makes the keratocyte-shape an ideal subject of modelling studies (Grimm et al., 2003; Rubinstein et al., 2005). The common aspects of actin-based protrusion suggest that the mechanisms underlying cell motility share some universal properties, and this will be of interest in our paper.

At the front of the cell is a thin sheet-like structure called the lamellipod (see Fig. 3). This structure is known to be rich in actin, the major cytoskeletal constituent, and largely devoid of organelles (Steinmetz et al., 1997; Cameron et al., 2001). The front edge of the cell is continuously projected forward, while the rear is retracted. In contrast to other motile cells, keratocyte adhesion to the substrate is relatively uniform (Lee and Jacobson, 1997).

We pose the following questions:

1. Can the interactions of the regulatory small G-proteins and actin explain how a cell polarizes and then retains its polarity?

2. How can cell polarization spontaneously lead to the formation of a leading edge and a trailing edge, and how can this give rise to cell movement?

3. Can a model based on these interactions explain how a cell maintains a regular shape as it moves?

4. Are these interactions sufficient to explain how a cell could respond to new external cues by turning and moving in a new direction?

5. Does a qualitative model of this sort work within the range of biologically reasonable parameter values?

6. Is it able to account for typical cell speeds, time and length scales?

We first introduce the reader to the known roles, distributions, and dynamics of the small G-proteins that are used in our model, and then formalize how we implement these dynamics in the model. We further develop this subject by introducing actin dynamics and cell protrusion, with their interactions and feedbacks. While describing the spatial model formalism, the combination of proteins 
and filaments with cell-shape and forces exerted on the membrane will become clear. We than analyze how the model behaves under a set of realistic parameter values gleaned from the literature. Finally, we subject the model to "in silico" experiments which we compare to "in vitro / in vivo" observations.

\subsection{Small G-proteins}

\subsubsection{Role of Rho GTPases in Cell Motility}

The rho family small G-proteins, Rac, Rho and Cdc42, control many cellular functions, including rates of actin filament polymerization, capping, and branching. Cdc42 is considered to be a central regulator of polarity in cells from such diverse organisms as humans and yeast (EtienneManneville, 2004); Rac is required for protrusive activity, lamellipodia and membrane ruffles; and Rho is necessary for the formation of stress fibers (actin-myosin filament bundles), formation and maintenance of focal adhesion complexes (integrin containing junctions that connect the stress fibers to the extracellular matrix), and contraction of the cell body.

The small G-proteins are molecular switches that cycle between inactive (GDP-bound) and active (GTP-bound) forms, and between the cytosol and the cell membrane (Takai et al., 2001). These two coupled cycles are regulated by three classes of proteins: GEFs (GDP/GTP exchange factors), GAPs (GTPase-activating proteins), and GDIs (GDP dissociation inhibitors). Dissociation of GDP from the GDP-bound form of a small G-protein is stimulated by GEFs. Hydrolysis of GTP to GDP is stimulated by various GAPs. The third class, GDIs, inhibit the dissociation of GDP and maintain the small G-proteins in an inactive form. The small G-proteins are only soluble in the cytosol when they are in a complex with GDI.

Active Rac and Cdc42 both stimulate actin polymerization by activating the actin related protein, Arp2/3 complex (Higgs and Pollard, 2000; Ridley, 2001b). (The Rac pathway is via the WAVE protein complex, whereas Cdc42 interacts with WASp and N-WASp.) Once activated by any of these, Arp $2 / 3$ complexes associate with existing filaments to form branches (at angles of $70^{\circ}$ ) with new barbed ends (Amann and Pollard, 2001). When a quiescent cell is exposed to an external signal (e.g. a chemoattractant), Arp2/3 activation leads to massive nucleation of new barbed ends at the leading edge of the lamellipod (Redmond and Zigmond, 1993; Mullins et al., 1997, 1998; Zebda et al., 2000; Suetsugu et al., 2002). Rac also upregulates the membrane phospholipid $\mathrm{PIP}_{2}$, known to protect actin barbed ends from capping. Cells can respond to a wide range of inputs, e.g. chemotactic signals, physical stress, and cell-cell contacts. These signals are transduced by different families of receptors that eventually recruit and activate a Cdc42-GEF (Etienne-Manneville, 2004). Consequently, Cdc42 acts as a "hub" in the small G-protein interaction network, through which information essential for cell orientation is funnelled.

Many insights about the specific roles of the small G-proteins have been obtained by studies using either dominant negative or constitutively active forms. Interestingly, what has been observed is that reduction or prevention of normal motility follows from both constitutively active or dominant negative expression of any one of these proteins (concerning Cdc42:Allen et al. (1998); Etienne-Manneville (2004); Rac: Hall (1998); Nobes and Hall (1999); Firtel and Chung (2000); Ridley (2001a); Tsuji et al. (2002); Meili and Firtel (2003); Rho: Kaibuchi et al. (1999); Nobes and Hall (1999)). This highlights the importance of the functional interaction of all three small 
G-proteins for cell motility, as opposed to an independent, direct effect of each one of them on the movement.

\subsubsection{Spatial Localization and Crosstalk of Rho GTPases}

Biochemical evidence, summarised here, points to spatial localization and crosstalk between the small G-proteins. Kraynov et al. (2000) found that in migrating Swiss 3 T3 fibroblasts, active Rac forms a gradient from front to back (and highest at the leading edge, at the sites of actin polymerization). Cdc42 also accumulates at the leading edge of a stimulated cell (Ridley, 2001b; Wittmann and Waterman-Storer, 2001; Falet et al., 2002). Following the kinetics of Cdc42 activation during individual protrusions, Nalbant et al. (2004) found a close correlation between extension and retraction of a lamellipod and the rise and fall of Cdc42 activation. In contrast, active Rho is predominantly found at trailing edge of moving cells (Xu et al., 2003), its levels being inversely correlated to the levels of active Rac in many types of cells (Caron, 2003). For this reason, it is believed that, in migrating cells, active Rho forms a gradient inverse to that of Rac and Cdc42 (Raftopoulou and Hall, 2004).

Ridley et al. (1992) gave the first report of the crosstalk of small G-proteins: they found that Rac is needed for Rho-mediated stress fibre formation and concluded that Rac activates Rho (See also Nobes and Hall (1995)). While Cdc42-injected cells give rise to Rac-dependent morphological changes, such as lamellipodia, Rac-null cells injected with Cdc42 do not exhibit lamellipodia - this is consistent with Cdc42 or its effectors activating Rac (Nobes and Hall, 1995). Cdc42 can lead to activation of Rac by allosteric regulation of a Rac-GEF, Cool-2 (Baird et al., 2005). Thus, currently the general picture is that Cdc42 activates Rac, which activates Rho (Matozaki et al., 2000). Evidence of mutual inhibition between Cdc42/Rac and Rho has also been found (Van Leeuwen et al., 1997; Sander et al., 1999; Firtel and Chung, 2000; Jiménez et al., 2000; Zondag et al., 2000; Caron, 2003).

\section{Modelling polarity and cell protrusion}

We here assemble a multi-level model for the roles of small G-proteins and actin cytoskeleton in cell motility. At the lowest molecular level, we use PDEs to describe the spatio-temporal kinetics of the small G-proteins within the cell. Next, we represent the assembly and disassembly of oriented actin filament density, and its regulation in the cell by small G-proteins. Finally, we use a latticebased approach to describe the cell as a whole, focusing on its shape, membrane fluctuations, polarity and velocity. As these levels are inextricably linked, a multilevel modelling approach, such as this, is essential to understanding the emergent dynamics that arises.

We first explain the motivation and derivation of the underlying dynamics of the small Gprotein crosstalk, and how these are to be interpreted within the spatial context of the model cell. In Section 2.3, we discuss the importance of orientation and directionality of the actin network in the protrusion of the cell. Finally, we explain the spatial modelling approach, based on the Cellular Potts Model (CPM), in Section 2.4.

Our aim is to assemble a (minimal set of) biologically plausible interactions consistent with polarization of a cell. To avoid later confusion, we note the working definitions of the following terms that will be used in describing model behaviour: bistability will here denote the existence of 
two stable equilibria in the ODE version of the model. By spatial bistability we mean that a spatial variant of the model exhibits zones of stable size, through which more or less constant concentration values are maintained. (In general, a given domain could contain multiple zones presenting two possible functional outcomes, determined by which proteins are dominant.) Polarity is used to denote spatial bistability with exactly two zones, where the spatial segregation can be associated with a unique front and a back. We will later show that spatial bistability can be obtained in parameter regimes that are not associated with bistability of the ODEs.

\subsection{Rac, Rho and Cdc42 interactions}

To describe the small G-protein interactions we follow the scheme presented by Giniger (2002), shown in Fig. 1, which is consistent with the biomolecular experiments outlined in Section 1.2.2. Due to the current lack of detailed knowledge on small G-protein crosstalk in keratocytes, and for the sake of being concise, we keep to this minimal set of well-established interactions that is consistently reoccurring throughout the many cell types studied. Recently, Sakumura et al. (2005) added variation to this underlying motif (adding an inhibition of Rac by Rho, and autoactivation of Rac), as well as a set of perturbations (deleting each of the interactions), to study possible oscillatory dynamics of these proteins in neuronal axon guidance. This exemplifies how one can further build upon this "core" description; a survey of the literature and detailed analysis of various possibilities appears in Jilkine (2005). Here, however, we seek to understand what these basic interactions are able to generate on their own.

The molecular interactions on which the model is based are depicted in Fig. 2; its derivation follows the same line as presented by Sakumura et al. (2005). We keep track of the small Gproteins $\mathrm{Cdc} 42$, Rac, and Rho that are active (on the membrane) or inactive (both on membrane and in cytosol, bound to GDI). $C, R$, and $\rho$ are defined as active, GTP-bound, Cdc42, Rac and Rho, respectively, while $C_{i}, R_{i}$, and $\rho_{i}$ are their inactive, GDP-bound, forms. All the variables are expressed in units of $[\mu \mathrm{M}]$. The fact that keratocyte fragments are also capable of manifesting similar movement is one indication that production and breakdown of small G-proteins does not play a role. Therefore only the cycling between active and inactive forms needs to be considered. The essential process of activation and deactivation through GEFs and GAPs is described as

$$
\frac{\partial G}{\partial t}=k_{G}^{+} \mathrm{GEF}_{G} G_{i}-k_{G}^{-} \mathrm{GAP}_{G} G+D_{m} \Delta G \quad \text { with } G=C, R, \rho,
$$

where $k_{G}^{+}$and $k_{G}^{-}$represent the kinetic rates of activation and inactivation, while $D_{m}$ represents the diffusion rate of the small G-proteins along the membrane, here taken to be the same for all three, based on their comparable molecular weight and structure. For constant total (active plus inactive) small G-protein, whose average level we define as $G_{t o t}=(1 /$ area $) \int\left(G+G_{i}\right) d A$, and for constant levels of the GAPs and GEFs in the absence of crosstalk $\left(\mathrm{GEF}_{\mathrm{G}}^{*}\right.$ and $\left.\mathrm{GAP}_{\mathrm{G}}^{*}\right)$, the above equation can be rewritten (by suitable redefinition of constants) as

$$
\frac{\partial G}{\partial t}=I_{G}\left(G_{i} / G_{t o t}\right)-d_{G} G+D_{m} \Delta G
$$

where $I_{G}=k_{G}^{+} \mathrm{GEF}_{G}^{*} G_{t o t}$ is a basal rate of GEF-mediated activation; and $d_{G}=k_{G}^{-} \mathrm{GAP}_{G}^{*}$ represents the basal inactivation rate mediated by GAPs; $G_{i} / G_{t o t}$ is a (dimensionless) local level of inactive 
small G-protein, scaled relative to the total amount of the given protein distributed in a well-mixed system. Thus, given the form of Eq. 2, the parameters $I_{G}$ and $d_{G}$ represent the basal activation and inactivation rates of a given small G-protein in the absence of the interactions with the others.

By now, molecular pathways have been determined for several specific examples of crosstalk between small G-proteins. From these, it has become clear that the activated form of these proteins elevate or suppress each other's activity by changing the activity of the GEFs or GAPs, either directly, or via a small cascade (Burridge and Wennerberg, 2004). Based on these findings, the crosstalk in our model is mediated by GEFs. This is similar to assumptions in Sakumura et al. (2005), who modelled these interactions through a combination of GAPs and GEFs; they used Michaelis-Menten saturating terms, which we choose to keep linear for the activation and sigmoidal for the inhibition. (See below). Due to this (in)activation of GEFs by the small G-proteins, the level of GEFs is no longer constant: We describe the upregulation of Rac by Cdc42 and Rho by Rac through a linear increase in GEF activation (see Fig. 2). Using basic QSS assumptions for the GEFs (which leads to $\mathrm{GEF}_{G}=\mathrm{GEF}_{G}{ }^{*}+k_{G^{\prime}} G^{\prime}$ ), the activating crosstalk interactions become

$$
\frac{\partial G}{\partial t}=\left(I_{G}+\alpha_{G^{\prime}} G^{\prime}\right)\left(G_{i} / G_{t o t}\right)-d_{G} G+D_{m} \Delta G, \quad \text { with } G=R, \rho ; G^{\prime}=C, R,
$$

where $\alpha_{G^{\prime}}$ describes the effect of the small G-proteins on activation of the GEFs $\left(\alpha_{G^{\prime}}=k_{G}^{+} k_{G^{\prime}} G_{t o t}\right)$.

For the description of the inhibiting interactions, we first observe that coupled inverted behaviour (flipping) is displayed by $\mathrm{Cdc} 42$ and Rho. We therefore use the toggle-switch model, derived by Gardner et al. (2000) for a similar biological system, to describe the mutual inhibition between Cdc42 and Rho, i.e. our model has terms representing cooperative inhibition of GEFs. We will show that cooperativity is essential for establishing a stable and strong spatial segregation. However, it is important to realize that a toggle switch by itself is not sufficient to explain sustained polarity.

The rate of reduced activation of protein $G$ under the influence of protein $G^{\prime}$ is then

$$
I_{G}\left(1-\frac{\left(G^{\prime}\right)^{n}}{\beta_{G^{\prime}}^{n}+\left(G^{\prime}\right)^{n}}\right)=\frac{I_{G}}{1+\left(G^{\prime} / \beta_{G^{\prime}}\right)^{n}} .
$$

Hence, to describe the inhibitory interactions, we write

$$
\frac{\partial G}{\partial t}=\frac{I_{G}}{1+\left(G^{\prime} / \beta_{G^{\prime}}\right)^{n}}\left(G_{i} / G_{t o t}\right)-d_{G} G+D_{m} \Delta G, \quad \text { with } G=C, \rho ; G^{\prime}=\rho, C,
$$

where $\beta_{G^{\prime}}$ is the level of small G-protein that leads to a half-maximal inhibition of the GEFs; $n$ describes the level of cooperativity.

Taken together, the above considerations lead to the set of equations for active GTP-bound Cdc42, Rac and Rho:

$$
\begin{aligned}
\frac{\partial C}{\partial t} & =\frac{I_{C}}{1+\left(\rho / \beta_{\rho}\right)^{n}}\left(C_{i} / C_{t o t}\right)-d_{C} C+D_{m} \Delta C, \\
\frac{\partial R}{\partial t} & =\left(I_{R}+\alpha_{C} C\right)\left(R_{i} / R_{t o t}\right)-d_{R} R+D_{m} \Delta R, \\
\frac{\partial \rho}{\partial t} & =\frac{\left(I_{\rho}+\alpha_{R} R\right)}{1+\left(C / \beta_{C}\right)^{n}}\left(\rho_{i} / \rho_{t o t}\right)-d_{\rho} \rho+D_{m} \Delta \rho .
\end{aligned}
$$


We will later refer to the kinetic terms in these equations as $f_{C}\left(C, \rho, C_{i}\right), f_{R}\left(C, R, R_{i}\right), f_{\rho}\left(C, R, \rho, \rho_{i}\right)$ (or simply as $f_{C}, f_{R}, f_{\rho}$ ).

An observation we made in constructing the model is that the relatively rapidly diffusing inactive forms of the small G-proteins (distributed in both membrane and cytosol) play an important role in spatial bistability. By simple bookkeeping, each inactive form of the small G-proteins satisfies an equation of the form

$$
\frac{\partial G_{i}}{\partial t}=-f_{G}+D_{m c} \Delta G_{i} \quad \text { where } G=C, R, \rho
$$

To derive this aggregate equation for the inactive small G-proteins, there are two scaling aspects to note. First, $G_{i}$ is the total of both inactive membrane bound $\left(G_{m}\right)$ and cytosolic $\left(G_{c}\right)$ small G-protein. Obviously, molecules in a membrane and contained within the cell volume come in different concentration dimensions, leading us to quantify these values together in the following manner: taking a 2D description of the cell (and assuming constant thickness throughout, see below), we consider all the variables as effective mean concentrations within a vertical column though the cell (see Fig. 3). Such an approach is straightforward: we know both the number of molecules contained within the vertical column and the column's volume (hexagonal base times column's height), hence the effective mean concentration. This approach holds an experimentally intuitive interpretation, for it is as if one slices a cell and counts all the specific molecules contained in this cell fragment with known volume (usually, however, this is done on a coarser per cell basis (Michaelson et al., 2001)). Because the lamellipod constitutes a major part of the cell, and given its approximately constant thickness, we ignore the nucleus-region which bulges up (its effective volume that permits small G-protein diffusion, however, being largely reduced due to the presence of organelles), and describe the cell as having constant height determined by the lamellipod.

Second, $D_{m c}$ is the effective diffusion rate of the inactive form, resulting from diffusion of the protein in both the membrane and the cytosol. The amount of cytosolic small G-protein, $G_{c}$, will vary due to exchange with the membrane,

$$
\frac{\partial G_{c}}{\partial t}=k_{o f f} G_{m}-k_{o n} G_{c}+D_{c} \Delta G_{i} \quad \text { where } G=C, R, \rho,
$$

with $k_{o f f}$ and $k_{o n}$ representing the rates of the molecule detaching from, and being incorporated into the membrane; $D_{c}$ is the diffusion rate in the cytosol. Recall that only the inactive form can detach from the membrane. (Due to the way the molecules of both forms are counted within the same column, as explained above, the dimensionality is already taken care of, i.e. $k_{o f f}$ and $k_{o n}$ both carry units [time $]^{-1}$.) It has been observed that the switching between both states is very rapid (Sako et al., 2000), allowing us to assume $G_{c}$ to be in QSS. From Eq. 9, it then follows that a fraction $k_{o n} /\left(k_{o n}+k_{o f f}\right)$ of $G_{i}$ is membrane-bound, and a fraction $k_{o f f} /\left(k_{o n}+k_{o f f}\right)$ is in the cytosol. Hence, the effective diffusion rate $D_{m c}$ is given by

$$
D_{m c}=\frac{k_{o n}}{k_{o n}+k_{o f f}} D_{m}+\frac{k_{o f f}}{k_{o n}+k_{o f f}} D_{c} .
$$

The value of the diffusion coefficient $D_{m c}$ should thus be in some range between the upper limit established by free diffusion of small G-proteins in the cytosol and the lower limit given by the membrane-bound diffusion, which is 10 to 100 -fold lower. 
All the concentration variables described above, i.e. $C, R, \rho, C_{i}, R_{i}, \rho_{i}$, depict proteins confined to the cell. We therefore have to use Von Neumann (or echo) boundary conditions along the edge of the cell.

\subsection{Arp2/3 dynamics}

We next model the concentration, $A$, of activated Arp2/3 complex, by the equation

$$
\frac{\partial A}{\partial t}=\mu_{C} C+\mu_{R} R-\eta\left(A, F_{t}\right) F_{t}-d_{A} A+D_{A} \Delta A,
$$

describing the activation of Arp2/3 by both Cdc42 and Rac (Ridley, 2001b) (via WAVE protein complex, respectively WASp and N-WASp), with rates given by $\mu_{C}$ and $\mu_{R}$. It then will be incorporated into the actin cytoskeleton network, at a rate that depends on both the Arp $2 / 3$ concentration and on the total amount of actin filaments, here represented by $F_{t}$ (see Eqn. (16) below). Note that this process does not depend on filament orientation. Further, Arp2/3 also decays and diffuses (with the rates $d_{A}$ and $D_{A}$, respectively). As above, $A$, is expressed in units of $[\mu \mathrm{M}]$, is confined to the cell, and satisfies Von Neumann (or echo) boundary conditions at the cell edge.

\subsection{Actin dynamics}

In our model, we keep track of actin filament density at various orientations. For their growth, we focus on the number of 'free' (uncapped) barbed ends that are able to extend. Levels of actin monomers are not considered as a limiting factor and the turnover rate of filaments is approximated as constant, i.e. we do not here distinguish between monomer-by-monomer depolymerization and other general filament degradation processes, nor do we take into account ageing (ATP-to-ADP decay) that makes the filament more prone to be broken down.

Actin filaments form a highly connected mesh, anchored to the substrate through focal adhesions. Because of the cross-linking of the cytoskeleton, we describe changes in actin density as being solely due to addition of monomers at uncapped barbed ends and to overall filament breakdown. This means that we neglect the small backward flow of cytoskeleton observed at the leading edge (Vallotton et al., 2004). The filament orientations dictate the local direction of extension of the cell. In keratocytes, most filaments near the leading edge point at angles within $\pm 70^{\circ}$ of the axis of protrusion (Maly and Borisy, 2001; Verkhovsky et al., 2003), resulting in forward translocation. Importantly, we do not impose a pre-determined cell front or back, nor do we make any ad hoc assumptions about local polarity information within the cell to guide the assembly of the network: rather, our purpose is to show that the observed orientation of the network can emerge as a natural consequence of the mechanism of protrusion.

To link the filaments to a hexagonal grid adopted for our lattice-based simulations, we keep track of six possible filament orientations, at discretized angles

$$
\Theta_{m}=\frac{2 \pi m}{6}
$$

This is a suitable description for Arp $2 / 3$ branching at $\pm 70^{\circ}$, here approximated by $60^{\circ}$ angles. 
We define $F_{\Theta_{m}}, B_{\Theta_{m}}$ to be the density of filaments and barbed ends at angle $\Theta_{m}$ in a given position at time $t . F$ is filament length per unit area ( $F$ has units of $[\mathrm{nm}] /[\mathrm{nm}]^{2}=[\mathrm{nm}]^{-1}$ ); $B$ is number of barbed ends per unit area (units of $[\mathrm{nm}]^{-2}$.) Introducing the hexagonal angle discretization, Eqn. (12), the equations describing growth and disassembly of filaments and extension, nucleation, and capping of their ends are

$$
\begin{aligned}
& \frac{\partial F_{\Theta_{m}}}{\partial t}=v_{0} B_{\Theta_{m}}-d_{F} F_{\Theta_{m}} \\
& \frac{\partial B_{\Theta_{m}}}{\partial t}=-\nabla \cdot\left(B_{\Theta_{m}} \vec{V}\left(\Theta_{m}\right)\right)+\frac{1}{2} k \eta\left(A, F_{t}\right)\left(F_{\Theta_{m-1}}+F_{\Theta_{m+1}}\right)-\kappa(R) B_{\Theta_{m}}
\end{aligned}
$$

where

$$
\vec{V}=v_{0}\left(\begin{array}{c}
\cos \Theta_{m} \\
\sin \Theta_{m}
\end{array}\right)
$$

Here $v_{0}$ is net polymerization speed at barbed ends (assumed constant), $v B_{\Theta_{m}}$ is the resulting increase in filament density, and $d_{F}$ is the filament disassembly rate. The first term in Eqn. (14) describes the flux of barbed ends due to growth of filaments oriented at angle $\Theta_{m}$; the second term describes the Arp2/3-mediated filament side-branching at a rate $\eta\left(A, F_{t}\right)$ that depends on effective filament length available for Arp $2 / 3$ binding. Half of the side-branches from a filament of orientation $\Theta_{m \pm 1}$ will then point in direction $\Theta_{m} . k$ is a scaling parameter (see Section 3); and $\kappa(R)$ describes the capping rate, assumed to depend on the levels of Rac.

The total filament and barbed end densities (summed over all orientations at a given location) are given by

$$
F_{t}=\sum_{m=1}^{6} F_{\Theta_{m}} ; \quad B_{t}=\sum_{m=1}^{6} B_{\Theta_{m}} .
$$

Moreover, given that all dynamics are confined to the cell, barbed ends reaching the edge of the cell will encounter resistance to motion due to the membrane. This is described through the boundary conditions of the model. We introduce the special class of force-bearing barbed ends, defined as $P_{\Theta_{m}}$, the number of barbed ends per unit edge length (in units of $[\mathrm{nm}]^{-1}$ ) that are actually pushing the membrane at angle $\Theta_{m}$, at a given position and time $t$ (see, e.g. Mogilner and Edelstein-Keshet (2002)). The rate at which barbed ends close to the membrane are promoted to this class is the product of their density and the velocity component perpendicular to the membrane (by classical conservation). Here they accumulate until discounted by capping. Thus, the total number of 'pushing barbed ends' per unit membrane length, oriented at angle $\Theta_{m}$, satisfies the following dynamics:

$$
\frac{\partial P_{\Theta_{m}}}{\partial t}=B_{\Theta_{m}} \vec{V}\left(\Theta_{m}\right) \cdot \hat{n}-r \kappa(R) P_{\Theta_{m}}
$$

where $\hat{n}$ is the unit vector normal to the edge. The first term has units of $[\mathrm{nm}]^{-2} \cdot[\mathrm{nm}][\mathrm{s}]^{-1}=$ $[\mathrm{nm}]^{-1}[\mathrm{~s}]^{-1}$. The second term represents (Rac-dependent) capping, assumed to be reduced by some factor $r$, since barbed ends in a zone close to the edge of the cell are protected from capping (see Section 3 for estimates of this parameter). 
To complete this description, we must specify how nucleation and capping depend on factors such as Arp2/3 and Rac. The following reaction scheme describes the binding of Arp2/3 to filaments:

$$
A+l F_{t} \underset{k_{-1}}{\stackrel{k_{1}}{\rightleftharpoons}} C \stackrel{k_{2}}{\rightarrow} l F_{t}+k B .
$$

The terms $l F_{t}$ and $k B$ describe, respectively, the amount of filaments (expressed in equivalent units of $[\mu \mathrm{M}])$ to which one $\mu \mathrm{M}$ of Arp2/3 binds, and the number of barbed ends generated by this binding. The scaling parameter $l$ is introduced because Arp2/3 and filament density have different units $\left([\mu \mathrm{M}]\right.$ vs. $\left.[\mathrm{nm}]^{-1}\right)$; it represents the minimum distance between branch points along an actin filament. (See also Section 3.) Notice that in various regimes, both filament density and Arp2/3 can be limiting factors for nucleation. Hence, a simple Michaelis-Menten description does not suffice and a total Quasi-Steady State Approximation (tQSSA) (Borghans et al., 1996) should be applied. Setting

$$
K_{m}=\frac{k_{-1}+k_{2}}{k_{1}}, \quad \eta_{0}=l k_{2},
$$

the tQSSA approximation leads to a nucleation function that saturates with respect to both variables:

$$
\eta=\eta_{0} \frac{A}{K_{m}+A+l F_{t}}
$$

Here, $\eta_{0}$ gives the maximum nucleation rate, and $K_{m}$ is a saturation constant.

Rac is known to directly activate PIP $_{2}$, which then inhibits capping. We propose the following relationship between the rate of barbed end capping, $\kappa(R)$, and active Rac:

$$
\kappa(R)=\kappa_{\text {max }}-\kappa_{\text {rac }}\left(\frac{R}{K_{R}+R}\right)=\kappa_{\text {basal }}+\kappa_{\text {rac }}\left(\frac{1}{1+\frac{R}{K_{R}}}\right) .
$$

Here $\kappa_{\max }$ is the maximal capping rate, $\kappa_{\text {rac }}$ the Rac-related reduction in capping (and $\kappa_{\text {basal }}=$ $\left.\kappa_{\max }-\kappa_{\text {rac }}\right) . K_{R}$ is the level of Rac at which its inhibition is one half of its maximal inhibition.

\subsection{Cell protrusion}

To integrate the regulatory elements, cytoskeleton dynamics and deformations and motion of the cell, we use the Cellular Potts Model (CPM), a spatial grid-based formalism that allows for mesoscopic cell description (Glazier and Graner, 1993): a cell is defined over a region, composed of multiple, in our case hexagonal, lattice sites (see Fig. 3). The dynamics are based on the free energy minimization principle (Marion and Thornton, 1995; Landau and Lifshitz, 1976), and generated by means of Monte Carlo Simulations utilizing the Metropolis algorithm (Metropolis et al., 1953). Effectively, this means that cell motion comes about from the overall minimization of the energy of deformation and stretching of the membrane through stochastic fluctuations, in which the global and local forces upon a cell edge, related to the internal structure and chemistry of the cell, are resolved (Graner, 1993).

We consider such an energy-based model to be an ideal approach to study biological cells (see also Discussion). Capturing all forces acting on a cell membrane accurately presents forbidding 
technical challenges. To circumvent this, we use an alternate approach based on Hamilton's principle (Marion and Thornton, 1995). This approach is used in many physical problems of classical mechanics where it is inconvenient, or even impossible, to decompose the effects on a system into a finite set of discrete point forces. Here we apply the formalism to describe the effects of protrusion and contraction of the cell edge on the cell dynamics.

Since the lamellipod is very thin $(\approx 176 \mathrm{~nm}$, Abraham et al. (1999)), we model the dynamics in 2D, using the quasi-3D approach described in Section 2.1 (see also Fig. 3). The fact that adhesion of the keratocyte to the substrate during movement is sufficiently homogeneous to allow for constant, roughly uniform traction forces between the cell and the substrate (Lee and Jacobson, 1997; Galbraith and Sheetz, 1999), enables us to focus on the fluctuations of the cell membrane that lead to protrusion and retraction of the cell edge. As stated, the biological cell on the substrate is described as a set of lattice sites (pixels) on a hexagonal grid, sharing the same state, $\sigma_{c e l l}$, while the medium surrounding it is represented by another state, $\sigma_{\text {medium }}$. The initial shape of the cell is round. The formation of a leading edge emerges from the interplay between small G-protein interactions, cell contraction and filament extensions (and is not a priori specified).

At each Monte Carlo time step (MCS), a random sampling through the lattice is used to determine whether pixels change their state into the state of one of their randomly chosen neighbours. This change (denoted "copying") corresponds to small deformations of the membrane. During one MCS each site is considered for a possible state change once. In the classical CPM, to determine whether copying will occur, one calculates an energy function, Hamiltonian $(H)$,

$$
H=\sum J_{C M}+\lambda(a-\mathcal{A})^{2},
$$

where the sum is over neighbouring sites (up to the $3^{\text {rd }}$ order) of each pixel. Through the Hamiltonian, the dynamics of the cell are established by minimizing the free energy. (As a result, large expansion or compression in the cell size, as well as large bending fluctuations of the cell membrane are avoided). In this $2 \mathrm{D}$ model, $H$ is a function of the cell area and the boundary length (corresponding to volume and surface area in 3D). $J_{C M}$ is the coupling energy per boundary site between a cell site (state $\sigma_{\text {cell }}$ ) and an empty adjoining site (state $\sigma_{\text {medium }}$ ) (from which the perimeter energy of the whole cell can be calculated); $a$ is the actual cell area, $\mathcal{A}$ the target area, and $\lambda$ an 'inelasticity' constant that affects the constraint on cell area deformation.

Given the Hamiltonian, the energy of a certain configuration can be determined, and a 'copying' of a random neighbour to a site can be tested: the energies of the two configurations, before and after copying, are compared by computing the net change of energy, $\Delta H=H_{\text {after }}-H_{\text {before }}$. The 'copying' trial is then accepted with probability

$$
P=\left\{\begin{array}{cl}
1 & \text { if } \quad \Delta H<-H_{b}, \\
\exp \left(-\frac{\Delta H+H_{b}}{T}\right) & \text { if } \quad \Delta H \geq-H_{b},
\end{array}\right.
$$

where $H_{b}$ represents a yield - the ability of the membrane to resist a force (Marée and Hogeweg, 2001), and $T$ (simulation temperature) determines the fluctuations. The parameters $T$ and $H_{b}$ represent specific effects (to be described) in the model formalism. In Section 3.3 we show how their values can be empirically and quantitatively connected to physical aspects of biological cells. We do so by requiring that the model correctly incorporates the relationship between the speed of membrane protrusion and barbed end density at the membrane, based on a thermal ratchet 
mechanism (Mogilner and Oster, 1996a, 2003). Eqn. (23) (the Metropolis Algorithm) means that changes in state that result in lowering the energy by at least $H_{b}$ are always made, whereas other changes are made with a Boltzmann probability. If the energy were to increase much due to such a state change, that change becomes extremely improbable.

Our model cell is endowed with internal chemistry that alters probabilities of expansion/retraction, making some parts bulge out more easily while other parts tend to retract. We extend the standard CPM-Hamiltonian to take this into account. To incorporate the pushing of actin filament ends on the cell edge membrane and the Rho-dependent contraction of parts of the cell, the Hamiltonian becomes

$$
\begin{aligned}
& \Delta H^{\prime}=\Delta H-\sum_{m} P_{\theta_{m}}+\xi\left(\rho-\rho_{t h}\right) \text { when the cell extends, } \\
& \Delta H^{\prime}=\Delta H+\sum_{m} P_{\theta_{m}}-\xi\left(\rho-\rho_{t h}\right) \text { when the cell retracts. }
\end{aligned}
$$

Forces exerted by all barbed ends pushing against the membrane towards the empty site, are represented by the linear shift in the Hamiltonian, $\sum P_{\theta_{m}}$. Note the sign difference in this term where the cell protrudes (extends by one site) versus where the cell retracts by one site (and the barbed ends then resist backward motion of the membrane). See Fig. 4(b). The last term describes Rho's influence on the contraction at the back of a motile cell. As incorporated in the Hamiltonian, the effective Rho-dependent contraction forces make inward fluctuations more likely, and outward fluctuations less likely at parts of the cell edge where Rho exceeds a certain threshold level for contraction, (i.e. when $\rho>\rho_{t h}$ ). The value $1 / \xi$ represents the elevation in Rho concentration whose resulting contraction counterbalances the force of one barbed end per nm pushing against the membrane. In our simulations, we use the quantity $\Delta H^{\prime}$ in the general update scheme in place of $\Delta H$ in Eqn. (23). $H, H_{b}$, and $T$ carry the same units as $P$, i.e. $[\mathrm{nm}]^{-1}$.

\subsection{Numerical simulations}

We numerically evaluate the model on a 500 x 500 hexagonal grid with wrap-around (toroidal) boundary conditions. The characteristic length of each grid point is scaled to $100 \mathrm{~nm}$. The lattice and discretized PDEs use the same resolution. One Monte Carlo time step corresponds to $0.1 \mathrm{~s}$, and the same timestep is used to numerically integrate the PDEs. Diffusion processes were integrated using an explicit Euler method, with sufficiently small timesteps to guarantee numerical stability. To initialize polarity (e.g. from the right to the left through the cell), a spatial gradient in the value of $I_{C}$ is applied for a period corresponding to $10 \mathrm{~s}$. This initialization represents the effective primary response of the cell to some external graded stimulus. Because the effect of external chemicals generally leads to the activation of Cdc42-GEFs (Etienne-Manneville, 2004), we incorporate the external signal in our model directly as an increase of the value of the parameter $I_{C}$. As soon as polarity is established, we remove spatial biases, and the autonomous internal dynamics of the proteins takes over. To test the response of a polarized cell to a new chemotactic signal, we reestablish after a $5 \mathrm{~min}$. interval another, (much smaller) gradient in $I_{C}$, along a different orientation.

When a site at the membrane retracts (i.e. when a site previously occupied by the cell becomes medium), all filaments and barbed ends formerly at this position get pushed back, together with the edge of the cell. As a consequence, the filaments pile up at adjacent sites inside the cell corresponding to their directions of orientation. The barbed ends, however, now push against a newly modified interface. They therefore become load-bearing (pushing) barbed ends, and are counted 


\begin{tabular}{|c|l|l|l|}
\hline Parameter & meaning & values & units \\
\hline$C^{*}$ & typical level of active Cdc42 & 1 & $\mu \mathrm{M}$ \\
$R^{*}$ & typical level of active Rac & 3 & $\mu \mathrm{M}$ \\
$\rho^{*}$ & typical level of active Rho & 1.25 & $\mu \mathrm{M}$ \\
$C_{t o t}$ & total level of Cdc42 & 2.4 & $\mu \mathrm{M}$ \\
$R_{t o t}$ & total level of Rac & 7.5 & $\mu \mathrm{M}$ \\
$\rho_{t o t}$ & total level of Rho & 3.1 & $\mu \mathrm{M}$ \\
$I_{C}$ & Cdc42 activation input rate & 3.4 & $\mu \mathrm{M} \mathrm{s}^{-1}$ \\
$I_{R}$ & Rac activation input rate & 0.5 & $\mu \mathrm{M} \mathrm{s}^{-1}$ \\
$I_{\rho}$ & Rho activation input rate & 3.3 & $\mu \mathrm{Ms}^{-1}$ \\
$\beta_{\rho}$ & Rho level for half-max inhibition of Cdc42 & 1.25 & $\mu \mathrm{M}$ \\
$\beta_{C}$ & Cdc42 level for half-max inhibition of Rho & 1 & $\mu \mathrm{M}$ \\
$n$ & Hill coefficient of Cdc42-Rho mutual inhibition response & 3 & - \\
$\alpha_{C}$ & Cdc42-dependent Rac activation rate & 4.5 & $\mathrm{~s}^{-1}$ \\
$\alpha_{R}$ & Rac-dependent Rho activation rate & 0.3 & $\mathrm{~s}^{-1}$ \\
$d_{C}, d_{R}, d_{\rho}$ & decay rates of activated small G-proteins & 1 & $\mathrm{~s}^{-1}$ \\
$D_{m}$ & diffusion coefficient of active small G-proteins & $1 \cdot 10^{5}$ & $\mathrm{~nm}^{2} \mathrm{~s}^{-1}$ \\
$D_{m c}$ & diffusion coefficient of inactive small G-proteins & $1 \cdot 10^{7}$ & $\mathrm{~nm}^{2} \mathrm{~s}^{-1}$ \\
\hline
\end{tabular}

Table 1: Parameter estimates relevant to the small G-proteins and their interactions.

as such. Similarly, as a membrane element extends, all the barbed ends that were formerly pushing against this membrane cease to be in direct contact with the membrane, becoming 'ordinary' barbed ends (no longer force-bearing) within the cell. In this way, filaments and barbed ends are not lost or generated de novo when the membrane retracts or extends, and the build-up and release of internal forces are directly coupled to the cytoskeleton (see Fig. 4(b)).

\section{Parameter estimates}

Many values of parameters used in the model can be inferred from some cell lineages, but not necessarily from keratocyte data. Therefore, we have assembled parameter estimates based on a composite set of data from various cell types. As we later point out, the behaviour of the model is fairly robust in large regions of parameter space around our estimates, leading to confidence in the biological relevance of the results.

A summary of parameter values used in the model appears in Tables 1,2 and 3. Details of how we calculated these estimates is given in the Appendix. Here we provide the general scheme used in finding values of the model parameters.

\subsection{Rac, Rho, and Cdc42 interactions}

To our knowledge, the concentration of small G-proteins in keratocytes is unknown. Michaelson et al. (2001), however, used immunoblotting to measure the total amount of small G-proteins in 


\begin{tabular}{|c|l|l|l|}
\hline Parameter & meaning & values & units \\
\hline$A^{*}$ & typical Arp2/3 concentration & 2 & $\mu \mathrm{M}$ \\
$F^{*}$ & typical filament density & 0.278 & $\mathrm{~nm}^{-1}$ \\
$B^{*}$ & typical barbed end density & $1.7 \cdot 10^{-5}$ & $\mathrm{~nm}^{-2}$ \\
$P^{*}$ & typical edge density of barbed ends & 0.05 & $\mathrm{~nm}^{-1}$ \\
$\mu_{C}, \mu_{R}$ & Cdc42 and Rac-dependent Arp2/3 activation & 0.16 & $\mathrm{~s}^{-1}$ \\
$d_{A}$ & activated Arp2/3 decay rate & 0.1 & $\mathrm{~s}^{-1}$ \\
$D_{A}$ & diffusion coefficient of Arp2/3 & $1 \cdot 10^{6}$ & $\mathrm{~nm}^{2} \mathrm{~s}^{-1}$ \\
$\eta_{0}$ & Arp2/3 nucleation rate & 60 & $\mu \mathrm{M} \mathrm{nm} \mathrm{s}^{-1}$ \\
$K_{m}$ & saturation constant for Arp2/3 nucleation & 2 & $\mu \mathrm{M}$ \\
$l$ & scale factor converting units of $F$ to concentration & 255 & $\mu \mathrm{M} \mathrm{nm}^{-2}$ \\
$k$ & scale factor converting concentration to units of $B$ & $1.06 \cdot 10^{-4}$ & $\mathrm{~nm}^{-2} \mu \mathrm{M}^{-1}$ \\
$v_{0}$ & actin filament growth rate (free polymerization) & 500 & $\mathrm{~nm} \mathrm{~s}^{-1}$ \\
$d_{F}$ & actin filament turnover rate & 0.03 & $\mathrm{~s}-1$ \\
$\kappa_{m a x}$ & barbed end capping rate & 2.8 & $\mathrm{~s}^{-1}$ \\
$\kappa_{r a c}$ & max reduction of capping by Rac & 2.1 & $\mathrm{~s}^{-1}$ \\
$K_{R}$ & Rac level for half-max reduction of capping & 3 & $\mu \mathrm{M}$ \\
$r$ & reduction of capping close to the edge & 0.14 & - \\
& & & \\
\hline
\end{tabular}

Table 2: Parameter estimates relevant to actin dynamics.

\begin{tabular}{|c|l|l|l|}
\hline Parameter & meaning & values & units \\
\hline$\Delta x$ & grid step size & 100 & $\mathrm{~nm}$ \\
$\Delta t$ & Monte Carlo time step (MCS) & 0.1 & $\mathrm{~s}$ \\
$T$ & simulation "temperature" & 0.008 & $\mathrm{~nm}^{-1}$ \\
$H_{b}$ & membrane yield & 0.046 & $\mathrm{~nm}^{-1}$ \\
$J_{C M}$ & coupling energy per boundary site & $7.5 \cdot 10^{-4}$ & $\mathrm{~nm}^{-1}$ \\
$\lambda$ & inelasticity constant & $10^{-9}$ & $\mathrm{~nm}^{-3}$ \\
$\mathcal{A}$ & target area of the cell & $3 \cdot 10^{8}$ & $\mathrm{~nm}^{2}$ \\
$\rho_{t h}$ & Rho threshold for contraction & 1.25 & $\mu \mathrm{M}$ \\
$\xi$ & effect of Rho on contraction & 0.06 & $\mu \mathrm{M} \mathrm{nm}^{-1}$ \\
$w$ & renormalised membrane resistance & 0.05 & $\mathrm{~nm}^{-1}$ \\
\hline
\end{tabular}

Table 3: Parameter estimates relevant to the CPM computation. 
fibroblasts (Cos1 cells). Using their results, we calculated effective total concentrations, of $\rho_{t o t}$, $R_{t o t}$, and $C_{t o t}$. There are large differences in the estimates of the fraction of small G-proteins that are actually in the GTP-bound state, ranging from $3 \%$ up to $25 \%$ for a resting cell (Boukharov and Cohen, 1998; Benard et al., 1999; Fleming et al., 1996); with the activity level of Rac and Cdc42 becoming at least twice as high when the cell is stimulated (Kurokawa et al., 2004). Based on these numbers, we here assume that during keratocyte motion, the overall fraction in the GTP-boundstate at steady state is around $40 \%$, leading to our estimates of $C^{*}, R^{*}$, and $\rho^{*}$.

The diffusion rate of the small G-proteins has not been measured, probably because the proteins cycle on and off the membrane. However, the diffusion of membrane-bound Ras, a small GTPase with the same molecular weight, is around $1 \mu \mathrm{m}^{2} \mathrm{~s}^{-1}$ (Goodwin et al., 2005), while Postma and Van Haastert (2001); Postma et al. (2004) report that the diffusion coefficient of heterotrimeric G-proteins in the membrane is $0.1 \mu \mathrm{m}^{2} \mathrm{~s}^{-1}$, and in the cytosol $10-50 \mu \mathrm{m}^{2} / \mathrm{s}$, i.e. two orders of magnitude larger. We used these results to estimate the diffusion coefficients of all forms of the small G-proteins and Arp2/3. To estimate decay rates of the small G-proteins, we used the reported average membrane lifetime of an activated Rac molecule (Sako et al., 2000), and the GAP-stimulated GTP hydrolysis of Rho (Zhang and Zheng, 1998). Saturation terms and the threshold $\rho_{t h}$ were taken to be in the same range as the typical steady-state concentrations. Based on estimated decay rates and approximate steady state concentrations, we inferred approximate activation rates for the small G-proteins. Finally, we used a Hill-coefficient $n=3$ for the mutually inhibiting responsecurves in Eqns. (5) and (7) (but see also Section 4). Further details are given in the Appendix.

\subsection{Actin dynamics}

In Eqn. (20), the term $l F_{t}$ describes the amount of filamentous actin (expressed in equivalent units of $[\mu \mathrm{M}])$ to which one $\mu \mathrm{M}$ of Arp2/3 binds. We used the minimal distance observed between sidebranches along a single filament (Mullins et al., 1998; Svitkina and Borisy, 1999), the lamellipod thickness (Abraham et al., 1999), and the number of monomers per unit filament length, to determine $l$, which converts units of actin density into corresponding units of Arp $2 / 3$ concentration. In the projected area of the lamellipod (shown as top view in Fig. 3), we computed effective densities of actin filaments $\left[\mathrm{nm}^{-1}\right]$ using the appropriate conversions.

Actin turnover rate and typical Arp2/3 equilibrium concentration were based on Pollard et al. (2000); Higgs and Pollard (1999). Typical filament density was taken from Abraham et al. (1999), which was then used to calculate maximal nucleation rates and the remaining Arp2/3 parameters. The barbed end capping rate was based on Pollard et al. (2000), with a reduction close to the cell membrane. (See, e.g. Grimm et al. (2003); Mogilner and Edelstein-Keshet (2002)).

\subsection{Cell protrusion}

In a thermal ratchet driven by actin polymerization (Mogilner and Oster, 1996a, 2003), the relationship between the number of barbed ends at the membrane and the speed, $v$, of the lamellipodial protrusion is approximately (Mogilner and Edelstein-Keshet, 2002)

$$
v=v_{0} \exp (-w / b)
$$


where $v_{0}$ is the free polymerization speed, $b$ the density of barbed ends per unit length at the membrane, and $w$ the renormalised membrane resistance force per unit length $\left(w=F_{m} \delta / k_{B} T\right.$, where $F_{m}$ is the membrane resistance, $\delta$ the size of one monomer, and $k_{B} T \approx 4.1 \mathrm{pN} \mathrm{nm}$ is thermal energy). Given the contrasting natures of the different models, a direct one-to-one correspondence between the CPM parameters and those of this force-based equation does not exist. However, we can link these values by deriving a comparable relationship between the effective speed of protrusion and the density of barbed ends for our model.

In the Appendix, we use a simple one-dimensional situation similar to that considered in Mogilner and Oster (1996a); Mogilner and Edelstein-Keshet (2002), to derive an effective force-velocity relationship for protrusion speed based on the CPM Hamiltonian implementation (Eqn 24) of barbed ends pushing at the cell edge. Likewise neglecting capping and side-branching, and assuming that all barbed ends are directed towards the cell edge, we show that the the implicit description of the mean speed of protrusion that stems from the Hamiltonian formulation is

$$
v=\frac{\Delta x}{\Delta t} \exp \left(-H_{b} / T\right)\left(\exp \left(\frac{\left(1-v / v_{0}\right) b}{T}\right)-\exp \left(\frac{-\left(1-v / v_{0}\right) b}{T}\right)\right) .
$$

While not identical to Eqn. (25), this equation also describes a relationship between protrusion velocity and the number of barbed ends, but here the relationship is expressed in terms of the CPM parameters $T$ and $H_{b}$. By fitting this relationship to Eqn. (25) (for which the parameter values are well-established), we can obtain the optimal values for $T$ and $H_{b}$. With this choice of CPM parameters $\left(T=0.008 \mathrm{~nm}^{-1} ; H_{b}=0.046 \mathrm{~nm}^{-1}\right)$ we obtain an excellent empirical fit between the thermal ratchet force-velocity relationship of Eqn. (25) and our effective force-velocity relationship, Eqn. (26), as shown in Fig. 4(a). (Note that the fit is close over the whole range of biologically relevant barbed end density values, typically observed to be in the range of $0.05-0.25 \mathrm{~nm}^{-1}$ at the lamellipod edge, Abraham et al. (1999); Mogilner and Edelstein-Keshet (2002).) Accordingly, the CPM gracefully leads to a reasonable depiction of actin-based protrusion forces, through energy minimization: for the above values of $T$ and $H_{b}$ our model quantitatively describes the response of the cell membrane to any possible load of pushing barbed ends.

Other details of parameter values are described in the Appendix. Briefly, $J_{C M}$ is linked to the effects of cell shape on protrusion of the edge. (At lower values of $J_{C M}$ ruffles can be formed more easily, while at higher values, the cell remains more compact.) As biological data is not available, we experimented with a range of values, before settling on $J_{C M}=7.5 \cdot 10^{-4} \mathrm{~nm}^{-1}$. The value of $\lambda$ is related to the compressibility of the cell (through osmotic forces, etc.), but has a limited effect on the dynamics. We choose $\lambda=10^{-9} \mathrm{~nm}^{-3}$, to keep fluctuations in cell size within a few percent. We used the typical 2D area of spread of a moving keratocyte (Svitkina and Borisy, 1999) to identify the target area $\mathcal{A}$ of $3 \cdot 10^{8} \mathrm{~nm}^{2} \simeq 3 \cdot 10^{4}$ lattice sites, and took a round cell, with a diameter $20 \mu \mathrm{m}$ for the initial configuration.

\section{Results}

\subsection{Establishment of polarity}

Mutual inhibition between Rho and Cdc42 can lead to bistability: either Cdc42 is high (which leads to activation and elevation of Rac) and Rho levels are suppressed, or Rho is high and Cdc42 and 
Rac are low. Whether bistability occurs depends on the Hill-coefficient, $n$, in $f_{C}, f_{\rho}$. Bistability (of the ODEs) is mathematically possible only for $n>1$ (Jilkine, 2005). For the biologically realistic parameter values used in this study, bistability in the temporal dynamics only occurs for $n \geq 4$, a narrower parameter range. When $n=6$, the bistable region becomes very large. Figs. 5(a) and (b), show the bifurcation diagrams for the well-mixed system in the cases $n=3$ and $n=6$.

A bistable regime of small G-protein dynamics, however, does not automatically lead to persistent polarity in a cell. In general, a stable transition zone does not occur at all in a spatially distributed bistable system with values at $\pm \infty$ fixed to the distinct steady states solutions. For example, using singular perturbation theory, Nishiura (1991) showed that a travelling wave will sweep through the domain in a 1D setting. Since one or the other equilibrium takes over, no stable coexistence is possible. If small G-proteins were always on the membrane, and never released into the cytosol, (i.e. if locally the amount of inactive Cdc42 could be described as $C_{i}=C_{t o t}-C$ ), this type of transient bistability would occur in our model. Fig. 5(c) shows that when bistability does not exist, the system immediately jumps to the stable equilibrium, while Fig. 5(d) shows that with bistability, such a travelling wave sweeps through the system, implying that polarity can only be maintained for a limited amount of time, and is then lost. Moreover, such bistability would depend strongly on the speed of cell motion and the level of external signals: only in an extremely tight parameter range would polarity be maintained for a reasonably long time.

This transient behaviour changes dramatically when the rapid diffusion of the GDI-bound small G-proteins in the cytosol (two-fold faster than in the membrane) is taken into account. The rapid cytosolic diffusion means that differences in cytosolic concentrations equilibrate very rapidly. The exchange between the membrane and the cytosol is also rapid (Sako et al., 2000). This leads to the following results: the activation of each of the small G-proteins, and thus its growth is limited by the availability of the inactive form. High levels of the inactive forms in the cytosol indicate overall low levels of activity, which means that local concentrations of the inactive forms indicate global activity levels. Figs. 5(e) and (f) show that an equilibrium is rapidly established, where expansion of each of the small G-proteins is limited by the availability of the inactive form. Moreover, due to this global competition, the equilibrium values are actually more extreme (Figs. 5(g) and (h)).

The triggering of chemotactic movement, in general, takes place through Cdc42 activation (Etienne-Manneville, 2004). But for a cell to retain its sensitivity to external signals, it is essential that an increase in Cdc42 activation would not lead to loss of polarity to transients. Persistent polarity and motion necessitates a stable transition zone between high Cdc42 (at the front) and high Rho (at the back). We asked how the location of this transition zone in the antagonistic gradients of $\mathrm{Cdc} 42$ and Rho depends on the activation rate, $I_{C}$ of Cdc42. Using Rac, Rho, and Cdc42 interactions, in a reduced 1D model of a "cell" (of typical length scale, $20 \mu \mathrm{m}$, with von Neumann boundary conditions), we numerically established the location of the transition zone for values of $I_{C}$ between 0.0 and $50.0 \mu \mathrm{M} \mathrm{s}^{-1}$. Surprisingly, spatial bistability was found not only within the region expected from the well-mixed case $\left(2.5<I_{C}<6.5\right)$, but also extended to much higher values $\left(1.7<I_{C}<43.4\right)$, in the spatially explicit case (Fig. 5(j)). More surprisingly, even for a Hill-coefficient as low as $n=3$ (a value inconsistent with bistability in the well-mixed case) spatial bistability changes only marginally (Fig. 5(i)). This means that the fact that the system is very close to a cusp bifurcation can be exploited in the spatial case: the dynamics slows down due to the bifurcation nearby, so that a stable spatial pattern is obtained. When the system is too far away from the cusp (e.g. at $n=2$ ), this behaviour disappears again. In conclusion, the interactions 
between the small G-proteins generate a highly robust and stable polarized system, a system that is only marginally dependent on parameters, cell size and interaction strength.

\subsection{Keratocyte cell movement}

Movies of all the cell movement simulations can be obtained from http://theory.bio.uu.nl/stan/keratocyte.

When we run the full model with the biologically based parameter values and all variables initiated at their typical levels (Tables 1,2 and 3), with an initially unpolarized cell, we do not obtain spontaneous polarization without initially applying a external signal. This is in agreement with the experimental observations of Verkhovsky et al. (1999). Fig. 6(a) shows a contour-plot of the model keratocyte when no initial external stimulus is applied. Since the cell does not spontaneously polarize, neither leading nor trailing edge are formed. Consequently, without extension and retraction coupled to specific parts of the cell, the cell remains more or less fixed in space, and no motion occurs. Moreover, without any internal pattern formation taking place, the cell shape is round, consistent with the minimal energy configuration. This agrees with the observation that non-polarized stationary keratocyte fragments are symmetrically circular (Verkhovsky et al., 1999) and represents a "control" for our model.

If a gradient in Cdc42 signalling is applied during the first 10 s (100MCS), the cell rapidly polarizes, and, after removal of the signal, stabilizes with a front (high Rac and Cdc42) and a back (high Rho) (Fig. 6(b)). At the front, activation of Arp2/3 and prevention of capping leads to rapid filament extension and side-branching, causing the formation of a broad, smooth leading edge. Contraction in the back due to Rho leads to a ruffled trailing edge. Once the cell has established this shape, it moves at a speed of $90 \mathrm{~nm} / \mathrm{s}$, i.e. $5.4 \mu \mathrm{m} / \mathrm{min}$ (Fig. 6(c)). This is in the range of speeds of real moving keratocytes, although higher speeds have been observed. This somewhat lower speed is partly a consequence of the relatively low value of $v_{0}$ used here, as taken from Mogilner and Edelstein-Keshet (2002). (This is good agreement in light of the fact that no a posteriori fitting was done to parameters of the simulations to mimic observed cell motility.) The crescent shape of the moving keratocyte is stable over a long timespan, and the speed of motion is more or less constant. Moreover, the direction of motion is highly persistent, but on a longer timescale (of hours), the track smoothly deviates and the orientation becomes uncorrelated to its original value. With the addition of even very small variations in, for example, the Cdc42 GEFs activation rate, $I_{C}$ (which mediates external signals), cell turning behaviour occurs (see Section 4.4). Thus, in a more realistic, noisy environment, we expect that the persistence length of the cell motion would be shorter. (Note that the values of $I_{C}$ that change the orientation of the moving cell are not sufficient to polarize the initial, unpolarized cell.)

Motion of the model cell results from a fine balance between extension at the leading edge and retraction at the trailing edge. Because at this stage we could not relate the Rho induced contraction parameter, $\xi$, to experimental measurements, we determined its effect by numerical experiments. (See cell shape and dynamics in Fig. 7). When there is little or no contraction at the back (e.g. $\xi \leq 0.01 \mu \mathrm{M} \mathrm{nm}^{-1}$ ), the cell does not initiate translocation. However, its immobile shape is unstable, and, due to internal filament forces and polarization, the cell rotates around itself. At higher values of $\xi$ (e.g. $0.02 \leq \xi \leq 0.04$ ), the cell is able to move slowly, but the motion can be highly irregular, interspersed with sudden turns. At yet higher values $(0.05 \leq \xi \leq 0.07)$ motion 
becomes very regular, and the cell achieves the typical keratocyte shape. Further increasing $\xi$, however, makes contraction too dominant, leading to triangular-shaped cells.

\subsection{Internal architecture of the cell}

Fig. 9 shows the concentration profiles of the small G-proteins and Arp2/3. A sharp transition zone separating the front and back is clearly seen. Due to general curvature effects, a contour separating these regions is always a smooth curve. Since rapid diffusion of the inactive small G-proteins in the cytosol stabilizes both zones, the sizes of these regions remain more or less constant, even when the cell is moving (or turning) rapidly. Consequently, the demarcation line moves forward at the same speed as the cell protrusion speed.

As the cell moves forward, the filament mesh gradually 'shifts' into the region where most barbed ends are capped, Arp2/3 is depleted, and where, consequently, breakdown dominates. Figs. 10(a-c) show that barbed ends peak very close to the leading edge, and that the highest filament density is attained a few microns behind the edge, slowly dropping rearwards. Maximal filament density is on same order as in real keratocytes (up to a factor 2). Our predicted higher values are due to low capping rates in the simulated lamellipod wherever Rac is high and to our omission of 'ageing' and increased severing of filaments away from the leading edge.

For actual protrusion of the cell, the orientations of the filaments and barbed ends are much more important than their density. We did not assume any dependence on orientation for the processes of capping, polymerization, side-branching and severing. Even so, during the motion of our model keratocyte, we obtain a highly structured leading edge. In Figs. 10(d-e) we use a colour coding to depict orientation, density and isotropy of the filaments and barbed ends within the cell, while the histograms of Fig. 10(f) show the gross orientation within $1 \mu \mathrm{m}$ of the leading edge. The figures clearly show that in a broad region behind the leading edge, most filaments and barbed ends subtend angles within $\pm 60^{\circ}$ of the edge. This generates a strong, directed force that 'pushes' the edge forward (contrary to the case where filaments and barbed ends point in random directions). The development of this correct orientation can be understood as follows: Only the barbed ends pointing in the right direction will stay inside the protected region (with low capping and high nucleation) when they extend. All others will be left outside, to be capped and eliminated; see Maly and Borisy (2001) for a similar conclusion. Moreover, since daughter side-branches are formed at angles of $\pm 60^{\circ}$, some of these still point in the right direction by "inheritance". Both processes, together, lead to an orientation profile that is very comparable to the experimental situation. However, because we discretized angles into six possible orientations, our resolution limits our ability to study the precise details of the preferred orientation. (For example, we cannot explain the dominant angular preference around $\pm 30^{\circ}$; but see Mogilner and Oster (1996a); Maly and Borisy (2001).)

\subsection{Responses to external signals}

When an external signal is imposed on the moving cell, we observe a rapid reorganization, in which the internal structure adapts, leading the cell to turn, and re-orient along the direction of the new signal. Fig. 8 shows how shallow gradients in $I_{C}$, applied in a direction orthogonal to the cell's trajectory, suffice to provoke rapid turning, within minutes. The signal can be very weak: 
the smallest $I_{C}$ gradient which causes the cell to turn within a reasonable time (under $30 \mathrm{~min}$ ), is $10^{-6} \mu \mathrm{M} \mathrm{s}^{-1} \mathrm{~nm}^{-1}$, though lower values are still able to trigger a significant deviation towards the gradient. This gradient magnitude corresponds to $0.03 \mu \mathrm{Ms}^{-1}$ across a $30 \mu \mathrm{m}$ cell, i.e. as low as $1 \%$ over the whole diameter of the cell (and even smaller when the cell turns).

An emergent phenomenon seen here is that polarization (i.e. the spatial bistable state) sensitizes the cell. This can be explained as follows: while the cell is moving forward steadily, the total area dominated by each antagonist (Cdc42 vs. Rho) is roughly constant, and the border zone between their regions of dominance moves at the same speed as the leading edge. However, small differences in the local 'competition' between the equilibria can locally change the speed of propagation of the border zone. For example, if $\mathrm{Cdc} 42$ activation, $I_{C}$, is slightly higher at a certain location, it will reduce the speed of propagation of the Rho interface. However, rapid cytosolic diffusion of the inactive forms of the small G-proteins assures that the area of dominance of, for example, Rho is roughly conserved. This long-ranged effect makes the Rho-front at the opposite side of the cell move faster, to compensate for a local loss of area. Thus, a small increase in $I_{C}$ at one flank not only inhibits the propagation of the Rho interface there, but also enhances this same propagation at the opposite flank. This causes an increase of curvature of the interface: since diffusion smooths out sharp local discontinuities, the interface as a whole remains smooth and starts to rotate. Thus, the polarity of the cell, defined by the relative positions of $\mathrm{Cdc} 42$ and Rho regions of dominance, rotates as well. Actin dynamics respond to the new situation, leading to motion in a new direction. The specific manner in which the cell is polarized sets up the cell's ability to efficiently integrate and 'communicate' external information along the whole membrane, generating the rapid and directed responses with high sensitivity.

According to Parent and Devreotes (1999), theories to explain chemotaxis (e.g. in Dictyostelium discoideum) should account for high sensitivity (to gradients as low as $2 \%$ ), accuracy, directional sensing independent of motion, and also independent of the actin cytoskeleton. They suggested that the signal for directed motion resides upstream of actin regulators, and hypothesised that the small G-proteins play a key role. Our mechanism is in correspondence with all these observations.

\section{Discussion}

Lee A. Segel (2001): “. . . the goal of theoretical biologists should be to "compute an organism." This has now been done by Marée and Hogeweg (2001). The necessity of refining and generalizing the calculations and the importance of linking changing gene expression with cell movement means that this achievement is not the beginning of the end but rather the end of the beginning."

The above (and earlier) quote of Lee Segel, to whom this article is dedicated, are taken from his commentary on a model for cellular dynamics during culmination of the cellular slime mould. In summarizing the Cellular Potts Model formalism in this commentary, he emphasizes its strength and convenience, particularly in view of the level of complexity presented by cells. At the same time, he points to the role of this type of modelling in opening doors to integration of biological information, and challenges us to link experimental knowledge from multiple-levels into a "computable organism". Our "organism" here is the keratocyte cell. 
In recent years, contributions to the cell motility puzzle have been made in experimental research on the actin cytoskeleton, the small G-proteins, and many other factors. Assembling these pieces of the puzzle reveals a picture of complicated interactions, regulations and feedbacks at, and between, many different levels of organization. Formalizing this intricate web of knowledge into mathematical language allows one to integrate levels of organization, connect the pieces, and bridge gaps. It also leads to better understanding of the control processes related to polarity, locomotion, response to external signals, and other aspects of cell motility.

Previous theoretical work has focused on detailed models of specific processes. These include the thermal ratchet model for polymerization-dependent force (Mogilner and Oster, 1996a,b, 2003), filament side-branching (Carlsson, 2001, 2003), and filament-turnover (Mogilner and EdelsteinKeshet, 2002; Grimm et al., 2003). An overarching recent objective has been to start combining diverse aspects of cell motility into a framework of a spatial cell. Lauffenburger (1989); DiMilla et al. (1991); Gracheva and Othmer (2004) have studied one-dimensional cross-sections with predefined polarity, to analyse the role of cell-substrate adhesion, cytoskeleton force generation and actin-myosin. Introducing forces involved with protrusion and retraction together with filamentturnover, Bottino et al. (2002); Rubinstein et al. (2005) have developed 2D descriptions of a crawling nematode sperm cell and a lamellipod, respectively. Both models have convincingly revealed important aspects of cell motility. However, as it was not within the scope of their work to unravel the underlying regulation of the filament-turnover and cell polarity establishment, they introduced a predefined front and back (associated with specific properties) in their model cells.

Our goal here was to combine such modules into a keratocyte without assuming any predefined spatial information, nor specific functions associated to different regions of the cell. To achieve this, we incorporated the actin cytoskeleton in a spatial context, bridged to 'one level down' (regulation by the small G-proteins), and 'one level up' (the mesoscopic description of the cell). Our model makes the interactions between these levels explicit. Its predictions lead to insights on many aspects of cell motility: speed, shape, polarity, reorientation, sensitivity and robustness. We explicitly opted for a model structure in which terms and parameters can be directly linked to experimental observables. Our model was run with biologically-relevant parameter values, which allowed us to compare quantitatively model and experimental observables. The results turned out to be both qualitatively and quantitatively comparable.

\section{Cell shape and speed}

The simulated cell shape has a high degree of resemblance to that of a moving keratocyte. It also manifests similar constant motion, at comparable speeds. One of the outputs of our model is, indeed, the cell velocity (a quantity that arises from the integration of all levels of the model); another is the distribution of small G-proteins over the cell. Other qualitatively correct aspects that emerged were the distribution of filament orientations and the stable shape of the cell. What confers value to these simulation results, is that they emerged from realistic known parameters without fitting. This is largely due to the multilevel structure of the model and its feedbacks, and is linked to the robustness discussed further on.

To be able to include distinct levels of organization within one model, it was, in certain aspects, necessary to loosen the precision of some observables. It is important to keep in mind where exactly simplifications have been made (and also what complexity arises from combining these 
levels). For example, a continuous description of all possible angles of filament orientation was sacrificed (and replaced by hexagonal angle discretization) to attain a mesoscopic simulation of the cell, but with that shortcut we gained a description of cell dynamics and cell shape detail that can be compared to empirical observations. One of our important considerations was to preserve as faithful a spatial description as possible of cell characteristics such as shape and motion. We have seen here that spatial influences are important beyond a mere descriptive role, i.e. that they are fundamental in the inner workings of the mechanisms underlying polarity and gradient sensing: the way that processes at distinct levels are coupled is intimately linked with spatial distributions of signals, central controlling module of small G-proteins, and cytoskeletal dynamics.

This leads us to the question of why we adopted the given spatial formalism of the CPM for this study. Invariably, any choice implies simplifications of certain aspects of reality. Some approaches in the literature have been based on a more explicit description of effective forces, as for example, in Rubinstein et al. (2005). Importantly, one should realize that such "true forces" are also approximations, made by representing a continuum of forces by some small number of springs and dashpots. In each case, it is essential to understand the power and limitations of the approximations made. Generally, the force-based approach relies on the main assumption that the movement of the centre of mass of the cell, or of its subdivisions, can be adequately described by a limited number of forces acting upon or between them. This strategy is well suited to describing objects that undergo limited deformation (or to describe the dynamics of a macroscopic object). Here, we chose to represent different aspects of motile cells: first, the fact that they are highly deformable, second, that the movement of each position depends on the movement of the rest of the cell (e.g. via turgor pressure), third, that cell membrane dynamics is locally highly correlated, but only loosely correlated to centre of mass, and fourth, that fluctuations play an important role in the nature of cell protrusion and motility. To represent these aspects with explicit forces acting on each position of the membrane leads to a huge proliferation of variables, forces, and effects to be considered, and is prohibitive. We therefore opted for a statistical mechanics approach, stemming from condensed matter physics. Given the questions we pose here, this approach is adequate. Essentially, by computing changes in a Hamiltonian, local forces on the periphery of the cell are described implicitly (via energy gradients with respect to the position of each membrane element). The cell, by energetically exploring its configurational possibilities, is effectively being subjected to forces on a microscopic level. This level of complexity would otherwise be difficult to model.

Adhesion and traction forces between a keratocyte and the substrate on which it crawls are relatively uniform. Therefore, we describe only the deformation of shape that results from the protrusion and retraction of the edges of the cell. For a description of such fluctuations, the Cellular Potts Model is ideally suitable. CPM simulations naturally produce ruffling of the membrane with constant slight changes in the cell form. That is, even as the cell maintains the same overall shape and a constant global speed, it does not move as if under a simple translational force. Real cells are highly dissipative objects (given the internal structure within a microscopic scale): within a cell, viscosity dominates inertia ${ }^{1}$ (Graner, 1993). These characteristics favour a description of cell motion in terms of local energy gradients. However, it would be interesting to compare the predictions of our approach to simulation methods that integrate/simulate explicit forces.

${ }^{1}$ Its equation of motion would be $\frac{d \vec{r}}{d t}=\frac{1}{\mu}\left[-\vec{\nabla} E+\vec{F}_{i}\right]$, where $1 / \mu$ is cell motility, $\vec{r}$ is the position vector of the cell, and $\vec{F}_{i}$ are all possible forces acting on the cell, besides the energy field $E$ that it experiences (Graner, 1993). 
Biological studies have revealed the importance of stochastic events in the behaviour of cells. (For instance, even the simple membrane fusion of a vesicle is triggered by the binding of just a few calcium ions to a docking complex, a stochastic event that results in the incorporation of a whole new membrane fragment.) Because dynamics of a cell depend highly on fluctuations, a noteworthy feature of the CPM is indeed its control of fluctuations through the 'simulation temperature' (i.e. statistical effects) within the Monte Carlo simulations. Tuning the 'temperature' allows us to turn up or turn down the effect of stochastic fluctuations (of possibly different origin) in the model. For example, Mombach et al. (1995) have interpreted the CPM temperature as membrane fluctuation amplitude of cells, for comparison with effects of the drug cytochalasin-B (a suppressor of membrane ruffling).

\section{Polarity}

We find that the modelled cell can very rapidly and easily be polarized, after which a very stable pattern is established. Moreover, the spatial distribution of the small G-proteins coincides with experimental observations.

In this paper, we opted to represent the mutual interactions of small G-proteins using a six component dynamical system (active and inactive forms of the proteins, Cdc42, Rac, and Rho) that has bistable regimes of behaviour. We showed, however, that cell polarity occurs even outside of the bistable regime (e.g. when the Hill coefficient is $n=3$ ). This is an important observation, since pure bistability would lead to spurious effects. (For example, a cell could not be at rest: either Cdc42 or Rho would take over completely, leading to behaviour similar to a dominant positive Cdc42/Rac or Rho mutant cell.) Because our model works outside the bistable regime, a resting stable cell is possible. Due to the spatial feedbacks, stable polarity can be found in a very broad parameter regime. The concept of the "toggle switch", motivated by Gardner et al. (2000), is required to maintain the polarity. This has strong implications for experimental research, since as biochemical pathways underlying the small G-proteins are elucidated (Burridge and Wennerberg, 2004), one could search for the cooperativity that we postulate as essential.

On one hand, the small G-protein module could, in principle, be expanded in the future as more details and quantitative measurements arise. On the other hand, it is not necessarily beneficial to include all experimental information linked to cell motility at once: such strategy would obscure underlying principles by overwhelming us with details too quickly. The highly stable polarity is an example of complex behaviour resulting from few, (experimentally) well understood elements (i.e. an 'emergent property' of the dynamics). By limiting our model to the above well-established key players and processes we have demonstrated what these interactions can already generate, what they can explain, and in what they are incomplete.

Other theoretical explanations for polarity have been proposed (such as based on Turing diffusiondriven instability or involving upstream phosphoinositides, see, e.g. Meinhardt (2003); Narang (2006); Levchenko and Iglesias (2002); Gamba et al. (2005)). Such mechanisms should have to be subjected to the same scrutiny as ours. That is, one should ask of any competing theoretical mechanism, applied to whole cell movement simulations, whether it can, together with other known facts used here (a) give rise to stable shape and polarization in a 2D (or 3D) moving cell, (b) do so within the biologically relevant range of known parameter values for actin dynamics, (c) account for the sensitivity of the cell to new stimuli, and (d) predict reasonable mesoscopic outputs, such 
as cell speed and actin densities.

In our opinion, a mechanism based on Turing diffusion-driven instability would not easily replace ours here. First, time-scales involved for a Turing pattern to be established are too large, leading to much slower cell dynamics and responses to external stimuli. Second, Turing pattern depends greatly on spatial size: the behaviour of small fragments of keratocyte would be expected to differ greatly from that of larger (whole) cells. Finally, even if it were possible to somehow avoid these problems, there is still the technical issue that a Turing system requires very different rates of diffusion (e.g. for activator and inhibitor), while the small G-proteins have very similar molecular weights, and hence similar rates of diffusion, thus not satisfying Turing conditions.

Levchenko and Iglesias (2002); Gamba et al. (2005) have focused on the upstream processes which seem to be involved in polarization and gradient sensing. (One aspect of this process is the redistribution of proteins such as PTEN and PI3K that govern the regulation of phosphoinositides, a module that is upstream of the small G-proteins, and not treated here directly.) It would be very interesting to extend our model with a module to further describe these interactions, so to infer what additional behaviour this would generate.

\section{Cell reorientation}

Once polarized, cells present the capacity of reorientating to external cues; this even occurs for gradients as small as $1 \%$ over the cell diameter. Besides this great sensitivity, the response times observed is extremely quick: within $3 \mathrm{~min}$. the cell has completed a right-angle turn, still maintaining its polarity. We emphasize, that in our model, cell turning is completely self-generating: we do not have to introduce any other artificial "rule" beyond the essential molecular scheme described herein. Therefore we have to see the reorientation behaviour, as emerging from the feedbacks and feedforwards between the multiple levels of organization.

Note that Sakumura et al. (2005) present basic core interactions that are equivalent to ours, with a number of small extensions. Yet, their model does not generate phenomena such as gradient sensing (see fig. 5C in their paper; in their case, turning behaviour is only possible through some step-wise winner-takes-direction method). This is due to the absence of diffusion of the small G-proteins in their effectively well-mixed systems. This illustrates that coupling between the different levels (spatial and temporal, proteins and cell) is essential to successfully unravel underlying principles.

Our proposed mechanism could be tested, for it clearly indicates that rotation of the Cdc42/Rho border zone inside the cell prompts cell turning to a new external cue. It would be interesting to experimentally verify or falsify this prediction.

\section{Robustness}

A curious underlying aspect that we have observed in diverse simulations is robustness at a number of distinct levels: (i) robustness of parameters: a broad range of parameters is capable of generating the global behaviours, seen most clearly in robustness of the polarization; (ii) robustness in cell shape: cells undergo rapid responses to strong external cues while approximately conserving their shape, and then immediately recover their basic shape; (iii) robustness in reorientation sensitivity: the simulated cell is equally sensitive to signals of different absolute values (up to a 50 fold vari- 
ation) as long as the gradient is similar. Moreover, a small gradient is sufficient; (iv) robustness to noise and stochasticity: a very orderly process takes place within the cell, despite the apparent frantic wiggling of the cell membrane and the translocation of the entire cell. Such robustness is specific for the mechanism of polarity proposed here. It is of utmost importance that a model for cell polarity can handle naturally occurring fluctuations. In contrast to all above, the model shows no robustness for deletions of elements in the model structure itself. This could often be more difficult to show by experiments, for a high level of redundancy is generally observed in real cells.

Not all cells behave like keratocytes even though the core of their underlying machinery seems to be largely the same. A large diversity of forms and dynamics characterises the repertoire of cells (for example, yeast, Dictyostelium, neutrophils, and neuron growth cones). This leads to the question of how these diverse phenotypes are generated, and what are common or disparate underlying aspects of the mechanism. Experimental observations further increase the relevance of such questions: Fashena et al. (2002) showed that keratocyte-like movements occur in MCF7 breast adenocarcinoma cells overexpressing HEF1 (a cas-family protein), with the formation of a large leading lamellipod, enhanced ruffling and pronounced trailing edge. Similar observations were made in human microvascular endothelial cell line, HMEC-1 (Kiosses et al., 1999; Fischer et al., 2003). More surprisingly, Asano et al. (2004) reported that the deletion of a single gene, amiB, from Dictyostelium cells, transforms this typically amoeboid cell to a crescent-shaped keratocyte-like cell with persistent movement and higher velocity. These results suggest that the basic keratocyte motility might be more general, with features in common with other cell types. Because our model was able to explain how a lamellipod is formed and polarization is maintained, a future goal now would be to address what are the relevant differences causing the observed spectrum of cell motility repertoires. The discussion about robustness further suggests that we are now working with a minimal core mechanism for cell motion, and that small extensions could bring us to other cell movement modes. This is yet another way of validating our model, though here through further in silico experiments.

\section{Acknowledgements}

A.F.M.M. was supported by the Research Counsel for Earth and Life Sciences (ALW) with financial aid from the Netherlands Organization for Scientific Research (NWO). A.J. and A.T.D were supported by a combination of funds from the Mathematics of Information Technology and Complex Systems (MITACS Canada) and a subcontract (to LEK) from the National Science Foundation (USA) grant (number DMS-0240770) to Anders Carlsson, Washington University, St Louis. L.E.K. was supported by an NSERC discovery grant. We thank F. Graner and P. Hogeweg for valuable discussions. 


\section{References}

M. Abercrombie. The Croonian lecture, 1978: the crawling movement of metazoan cells. Proc. $R$. Soc. Lond. Ser. B, 207:129-147, 1980.

V. C. Abraham, V. Krishnamurthi, D. L. Taylor, and F. Lanni. The actin-based nanomachine at the leading edge of migrating cells. Biophys. J., 77(3):1721-1732, September 1999.

W. E. Allen, D. Zicha, A. J. Ridley, and G. E. Jones. A role for Cdc42 in macrophage chemotaxis. J. Cell Biol., 141(5):1147-1157, June 1998.

K. J. Amann and T. D. Pollard. Direct real-time observation of actin filament branching mediated by Arp2/3 complex using total internal reflection fluorescence microscopy. Proc. Natl. Acad. Sci. U.S.A., 98(26):15009-15013, December 2001.

Y. Asano, T. Mizuno, T. Kon, A. Nagasaki, K. Sutoh, and T. Q. P. Uyeda. Keratocyte-like locomotion in amiB-null Dictyostelium cells. Cell Motil. Cytoskeleton, 59(1):17-27, September 2004.

D. Baird, Q. Feng, and R. A. Cerione. The Cool-2/ $\alpha$-Pix protein mediates a Cdc42-Rac signaling cascade. Curr. Biol., 15(1):1-10, January 2005.

V. Benard, B. P. Bohl, and G. M. Bokoch. Characterization of Rac and Cdc42 activation in chemoattractant-stimulated human neutrophils using a novel assay for active GTPases. J. Biol. Chem., 274(19):13198-13204, May 1999.

J. A. M. Borghans, R. J. De Boer, and L. A. Segel. Extending the quasi-steady state approximation by changing variables. Bull. Math. Biol., 58(1):43-63, January 1996.

D. Bottino, A. Mogilner, T. Roberts, M. Stewart, and G. Oster. How nematode sperm crawl. J. Cell Sci., 115(Pt 2):367-384, January 2002.

A. A. Boukharov and C. M. Cohen. Guanine nucleotide-dependent translocation of RhoA from cytosol to high affinity membrane binding sites in human erythrocytes. Biochem. J., 330:13911398, March 1998.

K. Burridge and K. Wennerberg. Rho and Rac take center stage. Cell, 116(2):167-179, January 2004.

L. A. Cameron, T. M. Svitkina, D. Vignjevic, J. A. Theriot, and G. G. Borisy. Dendritic organization of actin comet tails. Curr. Biol., 11(2):130-135, January 2001.

A. E. Carlsson. Growth of branched actin networks against obstacles. Biophys. J., 81(4):19071923, October 2001.

A. E. Carlsson. Growth velocities of branched actin networks. Biophys. J., 84(5):2907-2918, May 2003.

E. Caron. Rac signalling: a radical view. Nat. Cell Biol., 5(3):185-187, March 2003. 
L. P. Cramer, T. J. Mitchison, and J. A. Theriot. Actin-dependent motile forces and cell motility. Curr. Opin. Cell Biol., 6(1):82-86, February 1994.

P. A. DiMilla, K. Barbee, and D. A. Lauffenburger. Mathematical model for the effects of adhesion and mechanics on cell migration speed. Biophys. J., 60(1):15-37, July 1991.

M. U. Ehrengruber, D. A. Deranleau, and T. D. Coates. Shape oscillations of human neutrophil leukocytes: characterization and relationship to cell motility. J. Exp. Biol., 199(Pt 4):741-747, April 1996.

S. Etienne-Manneville. Cdc42 - the centre of polarity. J. Cell Sci., 117(Pt 8):1291-1300, March 2004.

H. Falet, K. M. Hoffmeister, R. Neujahr, J. E. Italiano, Jr, T. P. Stossel, F. S. Southwick, and J. H. Hartwig. Importance of free actin filament barbed ends for Arp $2 / 3$ complex function in platelets and fibroblasts. Proc. Natl. Acad. Sci. U.S.A., 99(26):16782-16787, December 2002.

S. J. Fashena, M. B. Einarson, G. M. O’Neill, C. Patriotis, and E. A. Golemis. Dissection of HEF1dependent functions in motility and transcriptional regulation. J. Cell Sci., 115(Pt 1):99-111, January 2002.

R. A. Firtel and C. Y. Chung. The molecular genetics of chemotaxis: sensing and responding to chemoattractant gradients. BioEssays, 22(7):603-615, July 2000.

R. S. Fischer, K. L. Fritz-Six, and V. M. Fowler. Pointed-end capping by tropomodulin3 negatively regulates endothelial cell motility. J. Cell Biol., 161(2):371-380, April 2003.

I. N. Fleming, C. M. Elliott, and J. H. Exton. Differential translocation of Rho family GTPases by lysophosphatidic acid, endothelin-1, and platelet-derived growth factor. J. Biol. Chem., 271 (51):33067-33073, December 1996.

S. Funamoto, R. Meili, S. Lee, L. Parry, and R. A. Firtel. Spatial and temporal regulation of 3phosphoinositides by PI 3-kinase and PTEN mediates chemotaxis. Cell, 109(5):611-623, May 2002.

C. G. Galbraith and M. P. Sheetz. Keratocytes pull with similar forces on their dorsal and ventral surfaces. J. Cell Biol., 147(6):1313-1324, December 1999.

A. Gamba, A. De Candia, S. Di Talia, A. Coniglio, F. Bussolino, and G. Serini. Diffusion-limited phase separation in eukaryotic chemotaxis. Proc. Natl. Acad. Sci. U.S.A., 102(47):16927-16932, November 2005.

T. S. Gardner, C. R. Cantor, and J. J. Collins. Construction of a genetic toggle switch in Escherichia coli. Nature, 403(6767):339-342, January 2000.

E. Giniger. How do Rho family GTPases direct axon growth and guidance? A proposal relating signaling pathways to growth cone mechanics. Differentiation, 70(8):385-396, October 2002. 
J. A. Glazier and F. Graner. Simulation of the differential adhesion driven rearrangement of biological cells. Phys. Rev. E, 47(3):2128-2154, March 1993.

J. S. Goodwin, K. R. Drake, C. L. Remmert, and A. K. Kenworthy. Ras diffusion is sensitive to plasma membrane viscosity. Biophys. J., 89(2):1398-1410, August 2005.

M. E. Gracheva and H. G. Othmer. A continuum model of motility in ameboid cells. Bull. Math. Biol., 66(1):167-193, January 2004.

F. Graner. Can surface adhesion drive cell-rearrangement? Part I: biological cell-sorting. J. theor. Biol., 164:455-476, 1993.

H. P. Grimm, A. B. Verkhovsky, A. Mogilner, and J.-J. Meister. Analysis of actin dynamics at the leading edge of crawling cells: implications for the shape of keratocyte lamellipodia. Eur. Biophys. J., 32(6):563-577, September 2003.

A. Hall. Rho GTPases and the actin cytoskeleton. Science, 279(5350):509-514, January 1998.

J. M. Haugh, F. Codazzi, M. Teruel, and T. Meyer. Spatial sensing in fibroblasts mediated by 3' phosphoinositides. J. Cell Biol., 151(6):1269-1280, December 2000.

H. N. Higgs and T. D. Pollard. Regulation of actin polymerization by Arp $2 / 3$ complex and WASp/Scar proteins. J. Biol. Chem., 274(46):32531-32534, November 1999.

H. N. Higgs and T. D. Pollard. Activation by Cdc42 and $\mathrm{PIP}_{2}$ of Wiskott-Aldrich syndrome protein (WASp) stimulates actin nucleation by Arp2/3 complex. J. Cell Biol., 150(6):1311-1320, September 2000.

M. Huang, C. Yang, D. A. Schafer, J. A. Cooper, H. N. Higgs, and S. H. Zigmond. Cdc42-induced actin filaments are protected from capping protein. Curr. Biol., 9(17):979-982, September 1999.

A. Jilkine. Modelling the interactions of small GTPases. Master's thesis, University of British Columbia, Canada, 2005.

C. Jiménez, R. A. Portela, M. Mellado, J. M. Rodríguez-Frade, J. Collard, A. Serrano, C. MartínezA, J. Avila, and A. C. Carrera. Role of the PI3K regulatory subunit in the control of actin organization and cell migration. J. Cell Biol., 151(2):249-262, October 2000.

K. Kaibuchi, S. Kuroda, and M. Amano. Regulation of the cytoskeleton and cell adhesion by the Rho family GTPases in mammalian cells. Annu. Rev. Biochem., 68:459-486, 1999.

W. B. Kiosses, R. H. Daniels, C. Otey, G. M. Bokoch, and M. A. Schwartz. A role for p21-activated kinase in endothelial cell migration. J. Cell Biol., 147(4):831-844, November 1999.

T. P. Kole, Y. Tseng, I. Jiang, J. L. Katz, and D. Wirtz. Intracellular mechanics of migrating fibroblasts. Mol. Biol. Cell, 16(1):328-338, January 2005.

V. S. Kraynov, C. Chamberlain, G. M. Bokoch, M. A. Schwartz, S. Slabaugh, and K. M. Hahn. Localized Rac activation dynamics visualized in living cells. Science, 290(5490):333-337, October 2000 . 
C. E. Krewson, S. W. Chung, W. G. Dai, and W. M. Saltzman. Cell-aggregation and neurite growth in gels of extracellular-matrix molecules. Biotechnol. Bioeng., 43(7):555-562, March 1994.

K. Kurokawa, R. E. Itoh, H. Yoshizaki, Y. O. T. Nakamura, and M. Matsuda. Coactivation of Rac1 and $\mathrm{Cdc} 42$ at lamellipodia and membrane ruffles induced by epidermal growth factor. Mol. Biol. Cell, 15(3):1003-1010, March 2004.

L. D. Landau and E. M. Lifshitz. Mechanics, volume 1 of Course of theoretical physics. Butterworth-Heinemann, Oxford, third edition, 1976.

D. A. Lauffenburger. A simple model for the effects of receptor-mediated cell-substratum adhesion on cell migration. Chem. Eng. Sci., 44(9):1903-1914, 1989.

V. M. Laurent, S. Kasas, A. Yersin, T. E. Schäffer, S. Catsicas, G. Dietler, A. B. Verkhovsky, and J.-J. Meister. Gradient of rigidity in the lamellipodia of migrating cells revealed by atomic force microscopy. Biophys. J., 89(1):667-675, July 2005.

J. Lee and K. Jacobson. The composition and dynamics of cell-substratum adhesions in locomoting fish keratocytes. J. Cell Sci., 110:2833-2844, November 1997.

A. Levchenko and P. A. Iglesias. Models of eukaryotic gradient sensing: application to chemotaxis of amoebae and neutrophils. Biophys. J., 82(1 Pt 1):50-63, January 2002.

I. V. Maly and G. G. Borisy. Self-organization of a propulsive actin network as an evolutionary process. Proc. Natl. Acad. Sci. U.S.A., 98(20):11324-11329, September 2001.

A. F. M. Marée and P. Hogeweg. How amoeboids self-organize into a fruiting body: multicellular coordination in Dictyostelium discoideum. Proc. Natl. Acad. Sci. U.S.A., 98(7):3879-3883, March 2001.

J. B. Marion and S. T. Thornton. Classical dynamics of particles and systems, chapter 7. Harcourt Brace, Fort Worth, fourth edition, 1995.

T. Matozaki, H. Nakanishi, and Y. Takai. Small G-protein networks: their crosstalk and signal cascades. Cell. Signal., 12(8):515-524, August 2000.

R. Meili and R. A. Firtel. Two poles and a compass. Cell, 114(2):153-156, July 2003.

H. Meinhardt. Complex pattern formation by a self-destabilization of established patterns: chemotactic orientation and phyllotaxis as examples. C. R. Biol., 326(2):223-237, February 2003.

N. Metropolis, A. E. Rosenbluth, M. N. Rosenbluth, A. H. Teller, and E. Teller. Equation of state calculations by fast computing machines. J. Chem. Phys., 21:1087-1092, 1953.

D. Michaelson, J. Silletti, G. Murphy, P. D’Eustachio, M. Rush, and M. R. Philips. Differential localization of Rho GTPases in live cells: regulation by hypervariable regions and RhoGDI binding. J. Cell Biol., 152(1):111-126, January 2001. 
A. Mogilner and L. Edelstein-Keshet. Regulation of actin dynamics in rapidly moving cells: a quantitative analysis. Biophys. J., 83(3):1237-1258, September 2002.

A. Mogilner and G. Oster. Cell motility driven by actin polymerization. Biophys. J., 71(6):30303045, December 1996a.

A. Mogilner and G. Oster. The physics of lamellipodial protrusion. Eur. Biophys. J., 25(1):47-53, 1996b.

A. Mogilner and G. Oster. Force generation by actin polymerization II: the elastic ratchet and tethered filaments. Biophys. J., 84(3):1591-1605, March 2003.

J. C. M. Mombach, J. A. Glazier, R. C. Raphael, and M. Zajac. Quantitative comparison between differential adhesion models and cell sorting in the presence and absence of fluctuations. Phys. Rev. Lett., 75(11):2244-2247, September 1995.

R. D. Mullins, J. A. Heuser, and T. D. Pollard. The interaction of Arp2/3 complex with actin: nucleation, high affinity pointed end capping, and formation of branching networks of filaments. Proc. Natl. Acad. Sci. U.S.A., 95(11):6181-6186, May 1998.

R. D. Mullins, W. F. Stafford, and T. D. Pollard. Structure, subunit topology, and actin-binding activity of the Arp2/3 complex from Acanthamoeba. J. Cell Biol., 136(2):331-343, January 1997.

P. Nalbant, L. Hodgson, V. Kraynov, A. Toutchkine, and K. M. Hahn. Activation of endogenous Cdc42 visualized in living cells. Science, 305(5690):1615-1619, September 2004.

A. Narang. Spontaneous polarization in eukaryotic gradient sensing: a mathematical model based on mutual inhibition of frontness and backness pathways. Accepted for publication in J. theor. Biol., 2006.

M. D. Neely and J. G. Nicholls. Electrical activity, growth cone motility and the cytoskeleton. $J$. Exp. Biol., 198(Pt 7):1433-1446, July 1995.

Y. Nishiura. Singular limit approach to stability and bifurcation for bistable reaction diffusionsystems. Rocky Mt. J. Math., 21(2):727-767, 1991.

C. D. Nobes and A. Hall. Rho, Rac, and Cdc42 GTPases regulate the assembly of multimolecular focal complexes associated with actin stress fibers, lamellipodia, and filopodia. Cell, 81(1): 53-62, April 1995.

C. D. Nobes and A. Hall. Rho GTPases control polarity, protrusion, and adhesion during cell movement. J. Cell Biol., 144(6):1235-1244, March 1999.

C. A. Parent and P. N. Devreotes. A cell's sense of direction. Science, 284(5415):765-770, April 1999.

T. D. Pollard, L. Blanchoin, and R. D. Mullins. Molecular mechanisms controlling actin filament dynamics in nonmuscle cells. Annu. Rev. Biophys. Biomol. Struct., 29:545-576, 2000. 
T. D. Pollard and G. G. Borisy. Cellular motility driven by assembly and disassembly of actin filaments. Cell, 112(4):453-465, February 2003.

M. Postma, L. Bosgraaf, H. M. Loovers, and P. J. M. Van Haastert. Chemotaxis: signalling modules join hands at front and tail. EMBO Rep., 5(1):35-40, January 2004.

M. Postma and P. J. M. Van Haastert. A diffusion-translocation model for gradient sensing by chemotactic cells. Biophys. J., 81(3):1314-1323, September 2001.

M. Raftopoulou and A. Hall. Cell migration: Rho GTPases lead the way. Dev. Biol., 265(1):23-32, January 2004.

R. A. Ream, J. A. Theriot, and G. N. Somero. Influences of thermal acclimation and acute temperature change on the motility of epithelial wound-healing cells (keratocytes) of tropical, temperate and Antarctic fish. J. Exp. Biol., 206(Pt 24):4539-4551, December 2003.

T. Redmond and S. H. Zigmond. Distribution of F-actin elongation sites in lysed polymorphonuclear leukocytes parallels the distribution of endogenous F-actin. Cell Motil. Cytoskeleton, 26(1): 7-18, 1993.

A. J. Ridley. Rho family proteins: coordinating cell responses. Trends Cell Biol., 11(12):471-477, December 2001a.

A. J. Ridley. Rho GTPases and cell migration. J. Cell Sci., 114(Pt 15):2713-2722, August 2001 b.

A. J. Ridley, H. F. Paterson, C. L. Johnston, D. Diekmann, and A. Hall. The small GTP-binding protein Rac regulates growth factor-induced membrane ruffling. Cell, 70(3):401-410, August 1992.

B. Rubinstein, K. Jacobson, and A. Mogilner. Multiscale two-dimensional modeling of a motile simple-shaped cell. SIAM Multiscale Model. Simul., 3(2):413-439, 2005.

Y. Sako, K. Hibino, T. Miyauchi, Y. Miyamoto, M. Ueda, and T. Yanagida. Single-molecule imaging of signaling molecules in living cells. Single Mol., 1(2):159-163, 2000.

Y. Sakumura, Y. Tsukada, N. Yamamoto, and S. Ishii. A molecular model for axon guidance based on cross talk between rho GTPases. Biophys. J., 89(2):812-822, August 2005.

E. E. Sander, J.-P. Ten Klooster, S. Van Delft, R. A. Van Der Kammen, and J. G. Collard. Rac downregulates Rho activity: reciprocal balance between both GTPases determines cellular morphology and migratory behavior. J. Cell Biol., 147(5):1009-1022, November 1999.

L. A. Segel. Computing an organism. Proc. Natl. Acad. Sci. U.S.A., 98(7):3639-3640, March 2001.

M. O. Steinmetz, D. Stoffler, A. Hoenger, A. Bremer, and U. Aebi. Actin: from cell biology to atomic detail. J. Struct. Biol., 119(3):295-320, August 1997. 
S. Suetsugu, H. Miki, and T. Takenawa. Spatial and temporal regulation of actin polymerization for cytoskeleton formation through Arp2/3 complex and WASP/WAVE proteins. Cell Motil. Cytoskeleton, 51(3):113-122, March 2002.

T. M. Svitkina and G. G. Borisy. Arp2/3 complex and actin depolymerizing factor/cofilin in dendritic organization and treadmilling of actin filament array in lamellipodia. J. Cell Biol., 145(5): 1009-1026, May 1999.

T. M. Svitkina, A. B. Verkhovsky, K. M. McQuade, and G. G. Borisy. Analysis of the actin-myosin II system in fish epidermal keratocytes: mechanism of cell body translocation. J. Cell Biol., 139 (2):397-415, October 1997.

Y. Takai, T. Sasaki, and T. Matozaki. Small GTP-binding proteins. Physiol. Rev., 81(1):153-208, January 2001.

T. Takubo and N. Tatsumi. Distribution of myosin and actin in moving human neutrophils detected by double-fluorescence staining. Anal. Quant. Cytol. Histol., 19(3):233-238, June 1997.

T. Tsuji, T. Ishizaki, M. Okamoto, C. Higashida, K. Kimura, T. Furuyashiki, Y. Arakawa, R. B. Birge, T. Nakamoto, H. Hirai, and S. Narumiya. ROCK and mDia1 antagonize in Rho-dependent Rac activation in Swiss 3T3 fibroblasts. J. Cell Biol., 157(5):819-830, May 2002.

P. Vallotton, S. L. Gupton, C. M. Waterman-Storer, and G. Danuser. Simultaneous mapping of filamentous actin flow and turnover in migrating cells by quantitative fluorescent speckle microscopy. Proc. Natl. Acad. Sci. U.S.A., 101(26):9660-9665, June 2004.

F. N. Van Leeuwen, H. E. T. Kain, R. A. Van Der Kammen, F. Michiels, O. W. Kranenburg, and J. G. Collard. The guanine nucleotide exchange factor Tiam1 affects neuronal morphology; opposing roles for the small GTPases Rac and Rho. J. Cell Biol., 139(3):797-807, November 1997.

A. B. Verkhovsky, O. Y. Chaga, S. Schaub, T. M. Svitkina, J.-J. Meister, and G. G. Borisy. Orientational order of the lamellipodial actin network as demonstrated in living motile cells. Mol. Biol. Cell, 14(11):4667-4675, November 2003.

A. B. Verkhovsky, T. M. Svitkina, and G. G. Borisy. Self-polarization and directional motility of cytoplasm. Curr. Biol., 9(1):11-20, January 1999.

A. Wells, K. Gupta, P. Chang, S. Swindle, A. Glading, and H. Shiraha. Epidermal growth factor receptor-mediated motility in fibroblasts. Microsc. Res. Tech., 43(5):395-411, December 1998.

T. Wittmann and C. M. Waterman-Storer. Cell motility: can Rho GTPases and microtubules point the way? J. Cell Sci., 114(Pt 21):3795-3803, November 2001.

J. Xu, F. Wang, A. Van Keymeulen, P. Herzmark, A. Straight, K. Kelly, Y. Takuwa, N. Sugimoto, T. Mitchison, and H. R. Bourne. Divergent signals and cytoskeletal assemblies regulate selforganizing polarity in neutrophils. Cell, 114(2):201-214, July 2003. 
N. Zebda, O. Bernard, M. Bailly, S. Welti, D. S. Lawrence, and J. S. Condeelis. Phosphorylation of ADF/cofilin abolishes EGF-induced actin nucleation at the leading edge and subsequent lamellipod extension. J. Cell Biol., 151(5):1119-1128, November 2000.

B. Zhang and Y. Zheng. Regulation of RhoA GTP hydrolysis by the GTPase-activating proteins p190, p50RhoGAP, Bcr, and 3BP-1. Biochemistry, 37(15):5249-5257, April 1998.

G. C. M. Zondag, E. E. Evers, J.-P. Ten Klooster, L. Janssen, R. A. Van Der Kammen, and J. G. Collard. Oncogenic Ras downregulates Rac activity, which leads to increased Rho activity and epithelial-mesenchymal transition. J. Cell Biol., 149(4):775-782, May 2000. 


\section{A Appendix}

\section{A.1 Parameter estimates of Rac, Rho, and Cdc42 interactions}

According to Michaelson et al. (2001) (using immunoblotting in Cos1 cells), the total amount of small G-proteins is 34,82 , and $26 \mathrm{ng} / 10^{6}$ cells, for $\rho, R$, and $C$ respectively. Based on the molecular weight of the small G-proteins ( $21 \mathrm{kDa}$, which is $3.49 \cdot 10^{-11} \mathrm{ng}$ ), and approximating cells as $10 \mu \mathrm{m}$ diameter spheres, this corresponds, respectively, to $1.86,4.49$, and 1.42 times $10^{3}$ molecules $/ \mu \mathrm{m}^{3}$. Using Avogadro's number $\left(6.02 \cdot 10^{23}\right)$ it follows that $1 \mu \mathrm{M}$ equals $602 \mathrm{molecules} / \mu \mathrm{m}^{3}$, and therefore the effective total concentrations, i.e. $\rho_{t o t}, R_{t o t}$, and $C_{t o t}$, are 3.1, 7.5, and $2.4 \mu \mathrm{M}$. Based on estimates of the fraction of small G-proteins in the GTP-bound state (3\% up to 25\% for a resting cell) (Boukharov and Cohen, 1998; Benard et al., 1999; Fleming et al., 1996) and the doubling of Rac and Cdc42 activity upon stimulation (Kurokawa et al., 2004), we took the overall fraction in the GTP-bound-state at steady state to be around $40 \%$ (i.e. $C^{*} \simeq 1 ; R^{*} \simeq 3$; $\left.\rho^{*} \simeq 1.25 \mu \mathrm{M}\right)$.

We based diffusion coefficients on the estimate for Ras $\left(1 \mu m^{2} s^{-1}\right)$ (Goodwin et al., 2005), and on estimates for heterotrimeric G-proteins in the membrane $\left(0.1 \mu \mathrm{m}^{2} \mathrm{~s}^{-1}\right)$ and cytosol $\left(10-50 \mu \mathrm{m}^{2} \mathrm{~s}^{-1}\right)$ (Postma and Van Haastert, 2001; Postma et al., 2004). We used $D_{m}=1 \cdot 10^{5} \mathrm{~nm}^{2} \mathrm{~s}^{-1}$; and $D_{m c}=1 \cdot 10^{7} \mathrm{~nm}^{2} \mathrm{~s}^{-1}$. For the Arp2/3 complex, which has a much larger molecular weight (200 kDa, (Mullins et al., 1997)), we use $D_{A}=1 \cdot 10^{6} \mathrm{~nm}^{2} \mathrm{~s}^{-1}$.

The average membrane lifetime of an activated Rac molecule is $2 \mathrm{~s}$ (Sako et al., 2000), giving a decay rate of $0.5 \mathrm{~s}^{-1}$. GAP-stimulated GTP hydrolysis of Rho has been measured as $1.5 \mathrm{~s}^{-1}$ (Zhang and Zheng, 1998), so we took decay rates of the small G-proteins to be $d_{C}=d_{R}=d_{\rho}=1 \mathrm{~s}^{-1}$. We further assumed that all saturation terms are in the same range as the typical steady-state concentrations, i.e. $\beta_{\rho}=\rho^{*} ; \beta_{C}=$ $C^{*} ; K_{R}=R^{*}$; and $K_{m}=A^{*}$. By similar reasoning, we also take $\rho_{t h}=\rho^{*}$. Based on estimated decay rates and approximate steady state concentrations, we inferred approximate activation rates for the small Gproteins, obtaining, $I_{C}=3.4 \mu \mathrm{M} \mathrm{s}^{-1} ; I_{R}+1.0 \alpha_{C}=5.0 \mu \mathrm{M} \mathrm{s}^{-1}$; and $I_{\rho}+3.0 \alpha_{R}=4.2 \mu \mathrm{M} \mathrm{s}^{-1}$. In our simulations, we use $I_{R}=0.5 \mu \mathrm{M} \mathrm{s}^{-1} ; \alpha_{C}=4.5 \mathrm{~s}^{-1} ; I_{\rho}=3.3 \mu \mathrm{M} \mathrm{s}^{-1} ;$ and $\alpha_{R}=0.3 \mathrm{~s}^{-1}$, to describe a strong dependency of Rac on $\mathrm{Cdc} 42$, and a weaker dependency of Rho on Rac. Finally, the Hill-coefficient of the mutually inhibiting response-curves in Eqns. (5) and (7), is $n=3$ (see Section 4).

\section{A.2 Parameter estimates of actin dynamics}

$l F_{t}$ in Eqn. (20) is the amount of filamentous actin (in monomer units) to which one $\mu \mathrm{M}$ of Arp2/3 binds. A minimal distance of about $37 \mathrm{~nm}$ (13.7 monomers) has been observed between side-branches along a single filament (Mullins et al., 1998; Svitkina and Borisy, 1999). Thus Arp2/3 binds to actin roughly in the ratio of 1 Arp2/3 complex per 13.7 monomers (i.e. $1 \mu \mathrm{M} A$ binds to $13.7 \mu \mathrm{M} F_{t}$ in terms of "monomer equivalents").

In the equations for actin dynamics, we represented filament density in length per unit area (i.e. $F_{t}$ is expressed in $[\mathrm{nm}] /[\mathrm{nm}]^{2}=[\mathrm{nm}]^{-1}$ ), and this has to be converted to Arp2/3 concentration units of $[\mu \mathrm{M}]$. From the fact that $1 \mu \mathrm{M}$ of actin equals $6.02 \cdot 10^{-7}$ monomers $/ \mathrm{nm}^{3}$, and that the lamellipod is typically $176 \mathrm{~nm}$ thick (Abraham et al., 1999), it follows that in the projected area of the lamellipod (shown as top view in Fig. 3), $1 \mu \mathrm{M}$ of actin corresponds to $1.06 \cdot 10^{-4}$ monomers $/ \mathrm{nm}^{2}$. Since one monomer corresponds to $2.7 \mathrm{~nm}$ of filament, this is equivalent to $2.86 \cdot 10^{-4} \mathrm{~nm}^{-1}$. Restated the other way around, $1 \mathrm{~nm}^{-1}$ filament corresponds to $1 /\left(2.86 \cdot 10^{-4}\right)=3.49 \cdot 10^{3} \mu \mathrm{M}$. Thus, an appropriate scale factor that accomplishes the conversion is $l=3.49 \cdot 10^{3} / 13.7=255 \mu \mathrm{M} \mathrm{nm}$.

In the same way, when Arp2/3 binds to the filaments, a concentration of $A=1 \mu \mathrm{M}$ generates $1.06 \cdot 10^{-4}$ barbed ends $/ \mathrm{nm}^{2}$, which means that $k$ in scheme (18) and Eqn. (14) equals $1.06 \cdot 10^{-4} \mathrm{~nm}^{-2} \mu \mathrm{M}^{-1}$. A typical highest filament density within a keratocyte is $F^{*}=0.278 \mathrm{~nm}^{-1}$ (Abraham et al., 1999), while a typical fast 
actin filament turnover has a half-life of $23 \mathrm{~s}$, i.e. $d_{F}=0.03 \mathrm{~s}^{-1}$ (Pollard et al., 2000). At the position with the highest filament density, the polymerization must be balancing the decay. Hence, using Eqn. (13) with $v_{0}=500 \mathrm{~nm}^{-1}, F^{*}=0.278 \mathrm{~nm}^{-1}$, and $d_{F}=0.03 \mathrm{~s}^{-1}$, it can be derived that, locally, $B^{*}=1.7 \cdot 10^{-5}$. Since capping is a very rapid process, Eqn. (14) can be assumed to be in QSS. Ignoring the flux-term, this gives an estimation of the barbed end nucleation rate, $k \eta F^{*}=5 \cdot 10^{-5} \mathrm{~nm}^{-2} \mathrm{~s}^{-1}$, from which follows that $\eta=$ $1.6 \mu \mathrm{Mnms}^{-1}$. A typical Arp2/3 equilibrium concentration is $A^{*}=2 \mu \mathrm{M}$ (Higgs and Pollard, 1999). Using Eqn. (20) and the values $\eta=1.6 \mu \mathrm{Mnm} \mathrm{s}^{-1} ; A^{*}=2 \mu \mathrm{M} ; K_{m}=2 \mu \mathrm{M} ; l=255 \mu \mathrm{M} \mathrm{nm}$; and $F^{*}=0.278 \mathrm{~nm}^{-1}$, an expected maximum nucleation rate can be derived of $\eta_{0}=60 \mu \mathrm{Mnm} \mathrm{s}^{-1}$. These value can be used in Eqn. (11) to derive that $\mu_{C} C^{*}+\mu_{R} R^{*}-2 d_{A}=0.44$. We therefore choose $\mu_{C}=\mu_{R}=0.16 \mathrm{~s}^{-1}$, and a small non-specific decay of $d_{A}=0.1 \mathrm{~s}^{-1}$.

The barbed end capping rate is roughly $2.8 \mathrm{~s}^{-1}$ (Pollard et al., 2000), and is reduced to a quarter of this value by the action of PIP 2 (Huang et al., 1999; Pollard et al., 2000). We therefore use $\kappa_{\text {basal }}=0.7 \mathrm{~s}^{-1}$ and $\kappa_{r a c}=2.1 \mathrm{~s}^{-1}$, which gives a maximum capping rate, $\kappa_{\max }$, of $2.8 \mathrm{~s}^{-1}$. The reduction of capping close to the membrane (the rate being $0.1 \mathrm{~s}^{-1}$ at the membrane according to Grimm et al. (2003); Mogilner and Edelstein-Keshet (2002)) suggests that in Eqn. (17) $r=0.14$, which leads to a slowest capping rate at the (leading) edge of $0.1 \mathrm{~s}^{-1}$.

\section{A.3 Parameter estimates and derivation of edge protrusion}

To derive the empirical relationship between barbed end density and protrusion speed shown in Figure 4(a), we consider a simple one-dimensional (1D) case, which corresponds to a cell boundary that is flat, rather than curved. We assume that there is no capping or side-branching, and that all barbed ends are directed towards the cell edge. In our model, barbed ends either extend at their maximal polymerization speed ( $v_{0}$ in Eqns. (14) and (25)), if they are away from the edge, or they do not extend at all, when pushing against the edge. Now, suppose that a fraction $f$ of the barbed end population $b$ have not yet reached the cell edge, whereas a fraction $(1-f)$ is actively pushing against the membrane at the edge. The mean values of $\Delta H^{\prime}$ involved in extending and retracting of the cell are given by Eqns. (24); and, assuming that $\Delta H^{\prime} \geq-H_{b}$ (a reasonable constraint to prevent perpetual extension of the cell), the probabilities of the cell to extend, respectively retract, are, as given by Eqn. (23),

$$
P_{\text {extend }}=\exp \left(\frac{-H_{b}-(1-f) b}{T}\right) ; P_{\text {retract }}=\exp \left(\frac{-H_{b}+(1-f) b}{T}\right),
$$

which simply means that the $(1-f)$ pushing population favours extension $\left(P_{\text {extend }}\right)$ and disfavours retraction $\left(P_{\text {retract }}\right)$. The effective speed of cell protrusion is thus

$$
v=\frac{\Delta x}{\Delta t}\left(P_{\text {extend }}-P_{\text {retract }}\right)=\frac{\Delta x}{\Delta t} \exp \left(-\frac{H_{b}}{T}\right)\left(\exp \left(\frac{(1-f) b}{T}\right)-\exp \left(\frac{-(1-f) b}{T}\right)\right),
$$

where $\Delta x$ and $\Delta t$ are the space- and timestep corresponding to one MCS. In our model the mean speed of all barbed ends (i.e. of both extending and stalled $b$ ) is given by

$$
\frac{f b \cdot v_{0}+(1-f) b \cdot 0}{b}=f v_{0} .
$$

If barbed ends extend faster than the mean protrusion rate, the fraction $f$ not yet at the edge starts to decrease, which leads to a reduction in the mean speed of the barbed ends; concurrently the fraction of barbed ends that are pushing starts to increase, raising the protrusion speed of the cell. The opposite is true when the barbed ends extend at a slower rate. Consequently, the fraction $f$ of barbed ends that are not pushing against 
the edge and the cell protrusion speed reach an equilibrium, at which $f v_{0}=v$, or, equivalently, $f=v / v_{0}$. Combining this with Eqn. (28), leads to the desired result,

$$
v=\frac{\Delta x}{\Delta t} \exp \left(-H_{b} / T\right)\left(\exp \left(\frac{\left(1-v / v_{0}\right) b}{T}\right)-\exp \left(\frac{-\left(1-v / v_{0}\right) b}{T}\right)\right) .
$$

We used the following observations to select parameter values. For fast moving keratocytes, $v_{0}$ lies between 300 and $3000 \mathrm{~nm} \mathrm{~s}^{-1}$ (Pollard et al., 2000); as in Mogilner and Edelstein-Keshet (2002), we here use $500 \mathrm{~nm} \mathrm{~s}^{-1}$. $w$ has been estimated in Mogilner and Edelstein-Keshet (2002) to be $0.05 \mathrm{~nm}^{-1}$. According to Abraham et al. (1999); Mogilner and Edelstein-Keshet (2002), the density of barbed ends at the edge of the protruding lamellipod are $0.05-0.25 \mathrm{~nm}^{-1}$. The values $T=0.008 \mathrm{~nm}^{-1} ; H_{b}=0.046 \mathrm{~nm}^{-1}$ were selected by fitting Eqn. (30) to Eqn. (25), as shown in Fig. 4(a). We have not yet found a way to determine $J_{C M}$ directly from experimental data, since this value is linked to the effects of cell shape on protrusion of the edge, which has, until now, received little attention. We used $J_{C M}=7.5 \cdot 10^{-4} \mathrm{~nm}^{-1}$ after some trials. The compressibility parameter, $\lambda$ has a limited effect on the dynamics; the value $\lambda=10^{-9} \mathrm{~nm}^{-3}$, keeps cell size fluctuations reasonable. The total projected area of a moving keratocyte is around $30 \mu \mathrm{m}$ by $10 \mu \mathrm{m}$ (Svitkina and Borisy, 1999), leading to our choice of target area $\mathcal{A}$ of $3 \cdot 10^{8} \mathrm{~nm}^{2} \simeq 3 \cdot 10^{4}$ lattice sites. 


\section{Figures}

Figure 1: Interactions and feedbacks between the small G-proteins and their effects on the actin cytoskeleton included in this paper. Intermediate steps that are not explicitly modelled are given in brackets. The small G-protein interaction scheme is based on Giniger (2002).

Figure 2: Molecular details of the small G-protein dynamics. The small G-proteins are activated and deactivated through GEFs and GAPs. The active, GTP-bound forms of the small G-proteins elevate or suppress each other's activity by changing the activity of the GEFs.

Figure 3: Schematic diagram to explain our model of a moving keratocyte. The top view (2D projection of the cell onto the substrate) is discretized using a hexagonal lattice. Concentrations at positions $(x, y)$ are the total concentrations contained in columns of height $d z$, projected on hexagonal sites of area $d a$. (For simplification, we assume a constant thickness in the vertical direction.) Insets: Top right: the possible actin filament orientations, $\Theta_{m}, m=1 \ldots 6$, defined in the model; Centre right: the density of barbed ends $B_{\Theta_{m}}$ and filaments $F_{\Theta_{m}}$ at angle $\Theta_{m}$ (e.g. for $m=1)$, at any site $(x, y)$ in the cell interior; Bottom right: barbed end and filament density, and pushing barbed ends $P_{\Theta_{m}}$ at any site along the edge of the cell, at angle $\Theta_{m}$ (e.g. for $m=5$ ). Note that the model does not specify any leading edge a priori. The shape of the cell, and its leading edge and trailing edge arise spontaneously by self-organization in the simulations. 
Figure 4: Protrusion force-velocity in our model closely fits the thermal ratchet force-velocity curve. (a) The velocity of edge protrusion, $v$, as a function of the number of barbed ends, $b$, at the protruding edge of the cell. Thick line: the thermal ratchet relationship (Mogilner and Oster, 1996a), see Eqn. (25). Thin line: our CPM-based relationship, Eqn. (26), as derived in Appendix A.3. The CPM parameter values $T=0.008 \mathrm{~nm}^{-1}$ and $H_{b}=0.046 \mathrm{~nm}^{-1}$ result in a close empirical fit of our force-velocity curve to the thermal ratchet curve over the whole range of relevant barbed end densities at the edge. The parameter $H_{b}$ controls the sigmoidal aspect of the function, while $T$ determines the steepness of saturation. All other parameters are as given in Tables 2-3. (b) Schematic diagram of how filaments and barbed ends are extended or retracted in our 2D simulations when the edge of the cell moves out into an empty site, or retracts by one site.

Figure 5: Analysis of the dynamics of the small G-protein module showing regimes of bistability. Left panels: a shallow switch (Hill coefficient $n=3$ ) in the mutual suppression between Cdc 42 and Rho. Right panels: a steep switch $(n=6)$. Top row: Bifurcation diagrams (for a spatially homogeneous version of Eqns. (5), (6), (7), (8), i.e. with $D_{m}=D_{m c}=0$ ) showing the equilibrium Cdc42 concentration as a function of the basal Cdc42 activation rate $I_{C}$. (a) For $n<4$ there is no bistable region. (b) A large region of bistability is found for $n=6$, with either high levels of Cdc42 and Rac and low levels of Rho, or vice versa. (c) and (d): Space-time plots of the same module in the spatial setting of a 1D strip, $20 \mu \mathrm{m}$ wide, with the inactive forms described implicitly, i.e. $C_{i}=$ $C_{t o t}-C$. Subpanels show active Cdc42 (left), active Rho (right), with lightest colours for highest concentrations. In (c) the system immediately jumps to the unique equilibrium; in (d) the highCdc42 equilibrium expands at a constant rate, while the high Rho equilibrium retracts, preventing long-term differentiation between a front and a rear. (Note 3 orders of magnitude difference in timescales.) (e) and (f): Space-time plots of the model in a 1D spatial setting, with the inactive forms of the small G-proteins, and their rapid diffusion in the cytosol, described explicitly. The dynamics equilibrate quickly, leading to a stable front and back, for both $n=3$ (e) and $n=6$ (f). $(\mathrm{g})$ and $(\mathrm{h})$ : Profile of the equilibrium distributions of active and inactive Cdc42 and Rho. (i) and (j): The location of the border between the $\mathrm{Cdc} 42$ and the Rho dominance regions (here defined as the position where $C=C^{*}=1 \mu \mathrm{M}$ ) is shown, in relation to the value of $I_{C}$, the basal Cdc42 activation rate. The parameter region where spatial bistability occurs is much larger than in the well-mixed system, and only marginally different between $n=3$ (i) and $n=6$ (j). All parameters are as given in Tables 1-3.

Figure 6: Contour plots showing cell shape and relative position at 5 min intervals. (a): A model keratocyte at rest is typically round and essentially stationary. (b): A moving model keratocyte takes on a crescent shape, and undergoes persistent motion. (c) The speed of the centre of mass for the cells shown in (a) and (b). The moving cell has a nearly constant speed of $90 \mathrm{~nm} / \mathrm{s}$. (All Parameter values as in Tables 1-3). 
Figure 7: Contour plots showing cell shape and relative position $5 \mathrm{~min}$ intervals for a range of values of the contractility parameter, $0 \leq \xi \leq 8$ (in units of $10^{-2} \mu \mathrm{M} \mathrm{nm}^{-1}$ ), from top to bottom. In each row, darker colours represent earlier times. Note that for an excessively high value, such as $\xi=8$, the cell becomes triangular, and the direction of its motion becomes unstable. (Parameter values as in Tables 1-3).

Figure 8: Contour plots showing the relative position over time of a simulated chemotactic cell in a range of gradients, $10^{-5} \leq I_{C} \leq 10^{-7}$ (in units of in $\mu \mathrm{Ms}^{-1} \mathrm{~nm}^{-1}$ ) at $5 \mathrm{~min}$ intervals within each run. (Value of $I_{C}$ indicated next to each run. Darker colours represent earlier time points.) Even very small gradients in the level of Cdc42 signalling cause cells to rapidly reorient towards the gradient: when the variation in $I_{C}$, taken over the whole cell, is smaller than $1 \%$ (corresponding to a gradient of $10^{-6} \mu \mathrm{Ms}^{-1} \mathrm{~nm}^{-1}$ in a cell of $30 \mu \mathrm{m}$ ), the cell still displays chemotactic behaviour. Inset: details of the rapid turning of one cell in a $10 \%$ gradient at 30 s time steps. The cell rotates by 90 degrees over a 3 min interval. (Parameter values as in Tables 1-3)

Figure 9: Typical distributions of the small G-proteins and Arp2/3 inside the cell during motion. (a) Cdc42, (b) Rac, (c) Rho, showing relative concentrations inside the cell, from low (dark) to high (light) values. (d) Arp2/3 concentration. (e) Concentration profiles along a cross-section through the centre of the cell, from the trailing edge to the leading edge of the polarized cell.

Figure 10: Actin filament and barbed end distributions inside a moving simulated cell. Top row: Relative densities of (a) barbed ends and (b) filaments. (c): Barbed end and filament density profiles along a horizontal cross-section through the centre of the cell. Bottom row: Orientationdensity profile of the barbed ends (d) and filaments (e). The colour represents the local mean orientation, (the angle of the resultant vector of the sum of all barbed ends, i.e. $\arccos \left(\frac{\left(\sum_{m} \overrightarrow{B_{\Theta}}\right) \cdot \hat{n}}{\left|\sum_{m} \overrightarrow{B_{\Theta}}\right|}\right)$.) Colour wheel coding depicts directions, e.g. "red=East", "green=NorthWest", "blue=SouthWest", etc., through the full range of compass directions in a clockwise manner). The intensity (i.e. from black to red, etc.) depicts total magnitude of the density, i.e. $\sum_{m}\left|\overrightarrow{B_{\Theta}}\right|$. The saturation of the colours (i.e. from white via pastel colours to red, etc.) depicts the isotropy of the barbed end orientation, i.e. $\frac{\left|\sum_{m} \overrightarrow{B_{\Theta}}\right|}{\sum_{m}\left|\overrightarrow{B_{\Theta}}\right|}$. (f): Orientation profile within the first $1 \mu \mathrm{m}$ from the established leading edge. Filaments and barbed ends mainly point at an orientation within $\pm 60^{\circ}$ of the leading edge. 